\title{
Free oscillations of a fluid sphere in an infinite elastic medium and long-period volcanic earthquakes
}

\author{
Ataru Sakuraba ${ }^{1}$, Jun Oikawa $^{2}$, and Yuichi Imanishi ${ }^{3}$ \\ ${ }^{1}$ Department of Earth and Planetary Physics, University of Tokyo, Hongo 7-3-1, Bunkyo-ku, Tokyo 113-0033, Japan \\ ${ }^{2}$ Earthquake Research Institute, University of Tokyo, Yayoi 1-1-1, Bunkyo-ku, Tokyo 113-0032, Japan \\ ${ }^{3}$ Ocean Research Institute, University of Tokyo, Minamidai 1-15-1, Nakano-ku, Tokyo 164-8639, Japan
}

(Received July 12, 1999; Revised July 25, 2001; Accepted October 25, 2001)

\begin{abstract}
A source model of long-period volcanic earthquakes is presented. We consider that a fluid-filled spherical cavity surrounded by an infinite elastic medium is excited into resonance like the Earth's free oscillations. The eigenequation of this system is derived in a general manner, making use of the spherical harmonic and spherical Bessel expansions. The solution is given as a complex number; its real part is the eigenfrequency and the imaginary part represents the attenuation coefficient of the oscillation. The eigenmodes are classified into five groups: (1) the compressional modes in a fluid sphere, (2) the compressional modes in a solid medium, (3) the shear modes in a solid medium, (4) the Stoneley modes, and (5) the torsional modes. We apply them to the long-period volcanic earthquake observed at Asama volcano, Japan. Estimating the characteristic frequencies and attenuation coefficients of the observed vibrations and assuming that the primary component $(f=1.73 \mathrm{~Hz})$ corresponds to the fundamental translation mode of a fluid sphere as one of the compressional modes in fluid, we conclude that the resonator which is a spherical cavity of diameter $220 \mathrm{~m}$ filled with steam of temperature $500^{\circ} \mathrm{C}$ and pressure 170 atm is favorable.
\end{abstract}

\section{Introduction}

In several varieties of earthquakes observed in an active volcano, long-period volcanic earthquakes are uniquely distinguished from any other seismic events and have close relations to the structure under the volcano and its activity (McNutt, 1992; Chouet, 1996). This type of earthquake possesses very distinct features; its spectrum has a number of narrow peaks in the frequency domain and these characteristic frequencies are significantly low. One may call some of them "harmonic tremors" as their oscillations last very long time. A harmonic tremor is considered as a superposition of sustaining long-period events (Chouet, 1996). Figure 1 shows an example of the long-period volcanic earthquake observed at Asama volcano, Japan, to which we will apply our model in Section 4 of this paper. Its waveform resembles an exponentially damped sinusoid having a very low characteristic frequency $(f=1.73 \mathrm{~Hz})$ and lasts for more than one minute. This event may be called one of the "longcoda" volcanic earthquakes. Similar seismic events are also observed at, for example, Mt. Galeras (Chouet, 1996; Gómez and Roberto, 1997), Mt. Meakan-dake (Nishimura and Yamashita, 1982), and many other volcanoes in the world.

Many attempts have been made to account for the longperiod seismic events and mysterious vibrations of active volcanoes. For example, mechanisms based on the resonance of elastic waves in a fluid sphere (Crosson and Bame, 1985; Fujita et al., 1995), a fluid pipe (Ferrick et al., 1982;

Copy right (C) The Society of Geomagnetism and Earth, Planetary and Space Sciences (SGEPSS); The Seismological Society of Japan; The Volcanological Society of Japan; The Geodetic Society of Japan; The Japanese Society for Planetary Sciences.
Chouet, 1985) and a fluid-filled crack (Chouet, 1988) have been considered to deal with specific structures under volcanoes. Instead of the resonance effects, vibrations due to volcanic fluid flowing through a channel (Julian, 1994) and a path-effect intrinsic to near-surface local structures (Kedar et al., 1996) are considered as physical mechanisms.

This paper focuses on a model based on acoustic resonance of a fluid sphere, firstly, because a sphere is one of the simplest shapes in nature and, secondly, because a complete description of its characteristic frequencies has never presented. Relating problems have been already solved. For example, free oscillations of a fluid sphere in vacuum have been well studied in an attempt to understand the nature of the Earth's free oscillations (Aki and Richards, 1980). Another example is free oscillations of an infinite elastic body, including a vacuum spherical cavity, quoted in one of problems in Landau and Lifshitz (1986). In their formalism, the eigenfrequency of its radial mode is given as a complex number whose imaginary part stands for the attenuation (damping) coefficient of the oscillations.

Fujita et al. (1995) argued the eigenoscillation of a fluid sphere embedded in an infinite elastic body in detail and presented mathematical formulae. However, they are only concerned with the radial motions; other higher-order eigenmodes remain unknown. We generalize their approach to take three-dimensional motions of fluid and elastic media by expanding any physical quantity in spherical harmonics to resolve its horizontal structure and in spherical Bessel functions to resolve its radial structure. The eigenfrequencies are given in a similar manner to the case of the Earth's free oscillations, though they are not real numbers. 

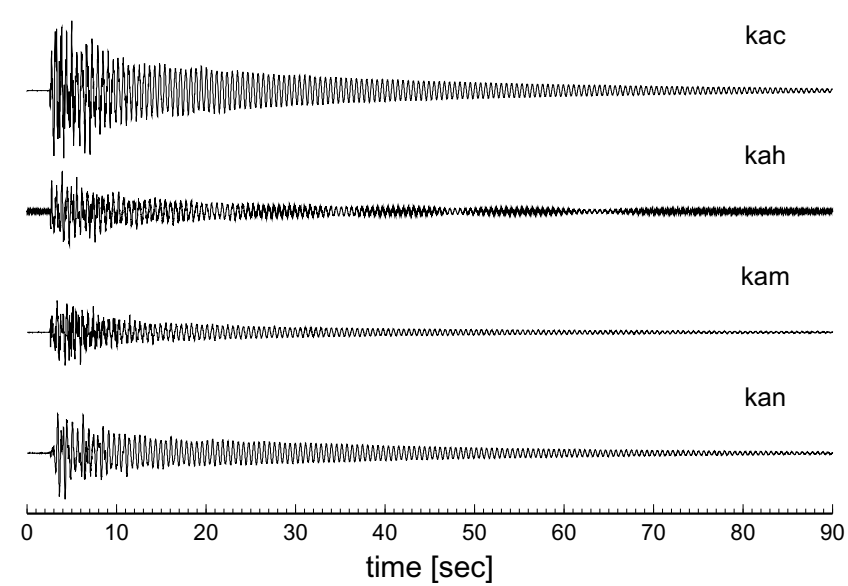

Fig. 1. Seismograms of the long-period event observed at four stations (KAC, KAH, KAM and KAN) around the summit of the Asama volcano. Vertical axis represents the relative magnitude of the up-down component of ground velocity.

In the following sections, we first present the general equations governing three-dimensional free oscillations of the system. Next, we describe the properties of the eigenmodes and classify them into several groups. The nature of vibrations is understandable as the combination of two special cases: free oscillations of a fluid sphere in vacuum and those of an infinite elastic body including a vacuum spherical cavity. However, some distinct features arise due to the interactions between fluid and solid media. The last part of this paper is devoted to the interpretation of the long-period volcanic earthquake observed at Asama volcano by applying our fluid-sphere model.

\section{Model}

Our model is essentially identical to that presented by Fujita et al. (1995). Let us consider a fluid sphere $M_{1}$ of radius $a$ which is embedded by a homogeneous elastic medium $M_{2}$ extending infinitely. We assume that the Lamé elastic parameters, $\lambda_{j}$ and $\mu_{j}$, and the density $\rho_{j}$ of $M_{j}$ are constants, where the subscript $j$ under a quantity indicates its value in the fluid sphere if $j=1$ and that in the solid medium if $j=2$ (Fig. 2). The $\mathrm{P}$ and $\mathrm{S}$ wave velocities are $\alpha_{j}=\sqrt{\left(\lambda_{j}+2 \mu_{j}\right) / \rho_{j}}$ and $\beta_{j}=\sqrt{\mu_{j} / \rho_{j}}$, respectively. Note that $\beta_{1}=0$, because there is no shear stress in the fluid sphere.

The equation governing elastic motion with no external forces can be written in the vector form

$$
\frac{\partial^{2} \boldsymbol{u}_{j}}{\partial t^{2}}=\alpha_{j}^{2} \nabla\left(\nabla \cdot \boldsymbol{u}_{j}\right)-\beta_{j}^{2} \nabla \times \nabla \times \boldsymbol{u}_{j},
$$

where $\boldsymbol{u}_{j}$ is the displacement in $M_{j}$. We decompose the vector field $\boldsymbol{u}_{j}$ into three terms:

$$
\boldsymbol{u}_{j}=\nabla F_{j}+\nabla \times \nabla \times\left(S_{j} \boldsymbol{r}\right)+\nabla \times\left(T_{j} \boldsymbol{r}\right),
$$

where $F_{j}, S_{j}$ and $T_{j}$ are the defining scalar functions and $\boldsymbol{r}$ is the position vector with respect to the center of the fluid sphere. In terms of the Earth's free oscillations, spheroidal oscillations are represented by $F_{j}$ and $S_{j}$, and torsional oscillations are represented by $T_{j}$. On substituting (2) into

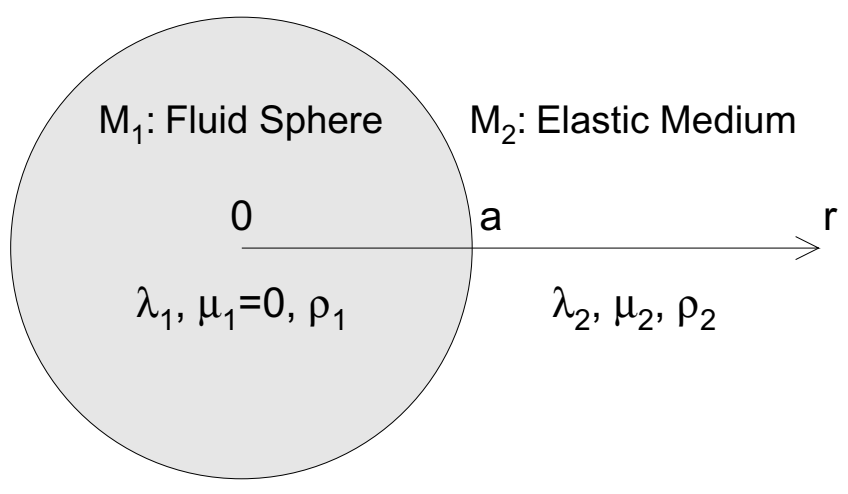

Fig. 2. A schematic view of the model.

Eq. (1), we have the following three independent equations describing general elastic motion:

$$
\begin{aligned}
& \frac{\partial^{2} F_{j}}{\partial t^{2}}=\alpha_{j}^{2} \nabla^{2} F_{j}, \\
& \frac{\partial^{2} S_{j}}{\partial t^{2}}=\beta_{j}^{2} \nabla^{2} S_{j}, \\
& \frac{\partial^{2} T_{j}}{\partial t^{2}}=\beta_{j}^{2} \nabla^{2} T_{j} .
\end{aligned}
$$

We assume that each defining scalar, say $F_{j}$, is expanded in the form

$$
F_{j}=\operatorname{Re}\left[\sum_{m=-\infty}^{\infty} \sum_{l=|m|}^{\infty} F_{j l}^{m}(r) Y_{l}^{m}(\theta, \phi) e^{i \omega t}\right],
$$

where $Y_{l}^{m}(\theta, \phi)=P_{l}^{m}(\cos \theta) e^{i m \phi}$ is the spherical harmonics, $P_{l}^{m}$ is the associated Legendre functions, $(r, \theta, \phi)$ are the spherical polar coordinates, and $\omega$ is the complex angular frequency. In this expression, the displacement and the traction across the spherical surface of radius $r$ are given by

$$
\begin{aligned}
u_{r}= & \operatorname{Re} \sum_{l, m}\left[\frac{d F_{l}^{m}}{d r}+\frac{l(l+1)}{r} S_{l}^{m}\right] Y_{l}^{m} e^{i \omega t}, \\
u_{\theta}= & \operatorname{Re} \sum_{l, m}\left[F_{l}^{m}+\frac{d}{d r}\left(r S_{l}^{m}\right)\right] \frac{1}{r} \frac{\partial Y_{l}^{m}}{\partial \theta} e^{i \omega t} \\
& +\operatorname{Re} \sum_{l, m} \frac{T_{l}^{m}}{\sin \theta} \frac{\partial Y_{l}^{m}}{\partial \phi} e^{i \omega t}, \\
u_{\phi}= & \operatorname{Re} \sum_{l, m}\left[F_{l}^{m}+\frac{d}{d r}\left(r S_{l}^{m}\right)\right] \frac{1}{r \sin \theta} \frac{\partial Y_{l}^{m}}{\partial \phi} e^{i \omega t} \\
& -\operatorname{Re} \sum_{l, m} T_{l}^{m} \frac{\partial Y_{l}^{m}}{\partial \theta} e^{i \omega t},
\end{aligned}
$$

$$
\begin{aligned}
\sigma_{r r}=\operatorname{Re} \sum_{l, m} & {\left[\lambda \mathcal{D}_{l}^{2} F_{l}^{m}\right.} \\
& \left.+2 \mu \frac{d}{d r}\left\{\frac{d F_{l}^{m}}{d r}+\frac{l(l+1)}{r} S_{l}^{m}\right\}\right] Y_{l}^{m} e^{i \omega t}, \\
\sigma_{r \theta}= & \operatorname{Re} \sum_{l, m} \mu\left[2 \frac{d}{d r}\left(\frac{F_{l}^{m}}{r}\right)+\frac{d^{2} S_{l}^{m}}{d r^{2}}\right.
\end{aligned}
$$




$$
\begin{gathered}
\left.+\frac{(l+2)(l-1)}{r^{2}} S_{l}^{m}\right] \frac{\partial Y_{l}^{m}}{\partial \theta} e^{i \omega t} \\
+\operatorname{Re} \sum_{l, m} \mu r \frac{d}{d r}\left(\frac{T_{l}^{m}}{r}\right) \frac{1}{\sin \theta} \frac{\partial Y_{l}^{m}}{\partial \phi} e^{i \omega t}, \\
\sigma_{r \phi}=\operatorname{Re} \sum_{l, m} \mu\left[2 \frac{d}{d r}\left(\frac{F_{l}^{m}}{r}\right)+\frac{d^{2} S_{l}^{m}}{d r^{2}}\right. \\
\left.+\frac{(l+2)(l-1)}{r^{2}} S_{l}^{m}\right] \frac{1}{\sin \theta} \frac{\partial Y_{l}^{m}}{\partial \phi} e^{i \omega t} \\
-\operatorname{Re} \sum_{l, m} \mu r \frac{d}{d r}\left(\frac{T_{l}^{m}}{r}\right) \frac{\partial Y_{l}^{m}}{\partial \theta} e^{i \omega t},
\end{gathered}
$$

where $\mathcal{D}_{l}^{2}$ is the differential operator defined by

$$
\mathcal{D}_{l}^{2}=\frac{d^{2}}{d r^{2}}+\frac{2}{r} \frac{d}{d r}-\frac{l(l+1)}{r^{2}},
$$

and the subscript $j$ is omitted for simplicity.

On integrating Eqs. (3)-(5) multiplied by $P_{l}^{m}(\cos \theta) e^{-i m \phi}$ on a unit sphere, we have

$$
\begin{aligned}
& \left(\mathcal{D}_{l}^{2}+p_{j}^{2}\right) F_{j l}^{m}=0, \\
& \left(\mathcal{D}_{l}^{2}+q_{j}^{2}\right) S_{j l}^{m}=0, \\
& \left(\mathcal{D}_{l}^{2}+q_{j}^{2}\right) T_{j l}^{m}=0,
\end{aligned}
$$

where $p_{j}=\omega / \alpha_{j}$ and $q_{j}=\omega / \beta_{j}$. Equations (13)-(15) are the Bessel differential equations of order of half an odd integer. Therefore, the solution is a linear combination of the spherical Bessel function $j_{l}$ and the spherical Neumann function $n_{l}$.

In the fluid sphere $M_{1}$, the defining scalars $S_{1}$ and $T_{1}$ must be zero because the $\mathrm{S}$ wave velocity $\beta_{1}$ is zero. Furthermore, the defining scalar $F_{1}$ is proportional to the spherical Bessel function to avoid singularities at the center of the sphere. Hence, the function $F_{1 l}^{m}(r)$ must be proportional to the spherical Bessel function of $l$ th order:

$$
F_{1 l}^{m}(r)=A_{l}^{m} j_{l}\left(p_{1} r\right),
$$

where $A_{l}^{m}$ is a complex constant. In the solid medium $M_{2}$, on the other hand, we adopt the radiative boundary condition at infinity which yields the solution of the outward spherical wave there. This condition is readily satisfied by forcing the functions $F_{2 l}^{m}(r), S_{2 l}^{m}(r)$ and $T_{2 l}^{m}(r)$ to be proportional to the spherical Hankel function of the second kind $h_{l}^{(2)}$ defined by

$$
h_{l}^{(2)}(z)=j_{l}(z)-i n_{l}(z)
$$

where $z$ is a complex variable. It is known that $h_{l}^{(2)}(z) \sim$ $i^{l+1} z^{-1} e^{-i z}$ in the limit of $|z| \rightarrow \infty$. Thus we have

$$
\begin{aligned}
F_{2 l}^{m}(r) & =B_{l}^{m} h_{l}^{(2)}\left(p_{2} r\right), \\
S_{2 l}^{m}(r) & =C_{l}^{m} h_{l}^{(2)}\left(q_{2} r\right), \\
T_{2 l}^{m}(r) & =D_{l}^{m} h_{l}^{(2)}\left(q_{2} r\right),
\end{aligned}
$$

where $B_{l}^{m}, C_{l}^{m}$ and $D_{l}^{m}$ are complex constants.

The boundary conditions at $r=a$ are the continuity of the radial component of displacement $u_{r}$ and the tractions across the boundary $\sigma_{r r}, \sigma_{r \theta}$ and $\sigma_{r \phi}$. Therefore, we have the following equations at $r=a$ :

$$
\begin{gathered}
A_{l}^{m} \frac{d}{d r} j_{l}\left(p_{1} r\right)=B_{l}^{m} \frac{d}{d r} h_{l}^{(2)}\left(p_{2} r\right) \\
+C_{l}^{m} \frac{l(l+1)}{r} h_{l}^{(2)}\left(q_{2} r\right), \\
A_{l}^{m} \lambda_{1} \mathcal{D}_{l}^{2} j_{l}\left(p_{1} r\right)=B_{l}^{m} \lambda_{2} \mathcal{D}_{l}^{2} h_{l}^{(2)}\left(p_{2} r\right) \\
+2 \mu_{2} \frac{d}{d r}\left[B_{l}^{m} \frac{d}{d r} h_{l}^{(2)}\left(p_{2} r\right)\right. \\
\left.+C_{l}^{m} \frac{l(l+1)}{r} h_{l}^{(2)}\left(q_{2} r\right)\right], \\
0=2 B_{l}^{m} \frac{d}{d r}\left(\frac{h_{l}^{(2)}\left(p_{2} r\right)}{r}\right) \\
+C_{l}^{m}\left[\frac{d^{2}}{d r^{2}}+\frac{(l+2)(l-1)}{r^{2}}\right] h_{l}^{(2)}\left(q_{2} r\right), \\
0=D_{l}^{m} \frac{d}{d r}\left(\frac{h_{l}^{(2)}\left(q_{2} r\right)}{r}\right) .
\end{gathered}
$$

The eigenfrequencies of the spheroidal oscillations are given by letting the determinant of the coefficient matrix of Eq. (21)-(23) be zero. Using the recurrence relations of the spherical Bessel function, we have the following characteristic equation:

$$
\left|\begin{array}{lll}
x_{1} & y_{1} & z_{1} \\
x_{2} & y_{2} & z_{2} \\
0 & y_{3} & z_{3}
\end{array}\right|=0
$$

where

$$
\begin{aligned}
x_{1} & =l j_{l}(\Omega)-\Omega j_{l+1}(\Omega), \\
x_{2} & =-R j_{l}(\Omega), \\
y_{1} & =l h_{l}^{(2)}(X)-X h_{l+1}^{(2)}(X), \\
y_{2} & =\left[2 l(l-1)-Y^{2}\right] h_{l}^{(2)}(X)+4 X h_{l+1}^{(2)}(X), \\
y_{3} & =2(l-1) h_{l}^{(2)}(X)-2 X h_{l+1}^{(2)}(X), \\
z_{1} & =l(l+1) h_{l}^{(2)}(Y), \\
z_{2} & =2 l(l+1)\left[(l-1) h_{l}^{(2)}(Y)-Y h_{l+1}^{(2)}(Y)\right], \\
z_{3} & =\left[2\left(l^{2}-1\right)-Y^{2}\right] h_{l}^{(2)}(Y)+2 Y h_{l+1}^{(2)}(Y), \\
X & =\Omega / \alpha, \\
Y & =X / \gamma, \\
R & =Y^{2} / \rho .
\end{aligned}
$$

Here, $\Omega=p_{1} a=\omega a / \alpha_{1}$ is the nondimensional angular frequency scaled with the characteristic time $\tau=a / \alpha_{1}$ and $\alpha, \gamma$ and $\rho$ are the nondimensional parameters defined by

$$
\alpha=\frac{\alpha_{2}}{\alpha_{1}}, \quad \gamma=\frac{\beta_{2}}{\alpha_{2}}, \quad \rho=\frac{\rho_{2}}{\rho_{1}} .
$$

The eigenfrequencies of the torsional oscillations are given by Eq. (24) as

$$
(l-1) h_{l}^{(2)}(Y)-Y h_{l+1}^{(2)}(Y)=0 .
$$


Note that the nondimensional eigenfrequencies $\Omega$ given by solving (25) or (27) discretely and infinitely exist in a complex plane. The real part of $\Omega$ represents the angular frequency of the free oscillations. The imaginary part, which is always positive, stands for the attenuation coefficient of the oscillations because the amplitude of the displacement is proportional to $e^{i \operatorname{Re}(\Omega) t-\operatorname{Im}(\Omega) t}$ at any place. The degeneracy with respect to the azimuthal wave number $m$ occurs like the case of free oscillations of a homogeneous sphere in vacuum. Hence, the eigenfrequency is generally written as $\Omega={ }_{n} \Omega_{l}$, where $n=0,1,2, \cdots$ is the number to identify the solution.

\section{Physical Properties of the Eigenmodes}

\subsection{Free oscillations of a fluid sphere in vacuum}

First of all, we describe the physical properties of the eigenmodes of two extreme cases. One extreme end is the free oscillations of a fluid sphere in vacuum, which is a special case with $\rho_{1}=0$ (i.e., $\rho=\infty$ ) in our model. The other is free oscillations of an infinite elastic body including a spherical void, which is another special case with $\rho_{2}=0$ (i.e., $\rho=0$ ).

The eigenfrequency ${ }_{n} \Omega_{l}$ of free oscillations of a fluid sphere in vacuum is given by the $(n+1)$ th root of the spherical Bessel function $j_{l}$ (see the left-hand side of Eq. (22) and also Aki and Richards (1980)). Note that ${ }_{n} \Omega_{l}$ is a real number because no emission of waves occurs in the present situation. Figure 3 shows the relation between the eigenfrequency $\Omega$ and the degree $l$; known as the dispersion relation of the eigenmodes. One can clearly see a number of branches of which the slope measures the group velocity of the resonating waves. We refer to these modes as "the compressional modes in a fluid sphere" and use the symbol ${ }_{n} S_{l}(n=0,1,2, \cdots)$ to indicate them because they are essentially the same as the Earth's spheroidal modes. The radial component of the displacement $u_{r}$ is shown in Fig. 4 for the eigenmodes ${ }_{0} S_{l},{ }_{1} S_{l}$ and ${ }_{2} S_{l}$. It is evident that the spherically symmetric (radial) modes ${ }_{n} S_{0}$ have $n$ nodes and the asymmetric modes ${ }_{n} S_{l}(l \geq 1)$ have $n+1$ nodes in the range $0<r<a$. The reason why there are no higherorder $(l \geq 1)$ eigenmodes which have no nodes in the range $0<r<a$, is that the shear stress is absent in the fluid sphere. It should be noticed that the use of the symbol ${ }_{n} S_{l}$ is different from that conventionally adopted in the case of the Earth's free oscillations. In Fig. 3, there exists an eigenmode ${ }_{n} S_{l}$ of $n=0$ and $l>0$. This does not mean that there is a higher-order mode having no nodes in the fluid region because $n$ does not represent the number of nodes but merely the index number to identify the branch.

\subsection{Free oscillations of a spherical cavity in an infinite elastic body}

The eigenfrequencies of the other extreme end, free oscillations of an elastic medium including a spherical cavity, are given by solving Eqs. (22), (23) and (24) in which $A_{l}^{m}=0$. The spheroidal modes (i.e., the solutions of (22) and (23)) are shown in Fig. 5 for $\gamma=0.5(\gamma$ is the ratio of the $\mathrm{S}$ and $\mathrm{P}$ wave velocities). In this figure, we temporarily defined a nondimensional angular frequency $\Omega^{\prime}=\omega a / \alpha_{2}$. One can find similar branches in the dispersion relation between $\operatorname{Re}\left(\Omega^{\prime}\right)$ and $l$, as seen in Fig. 3. These branches are

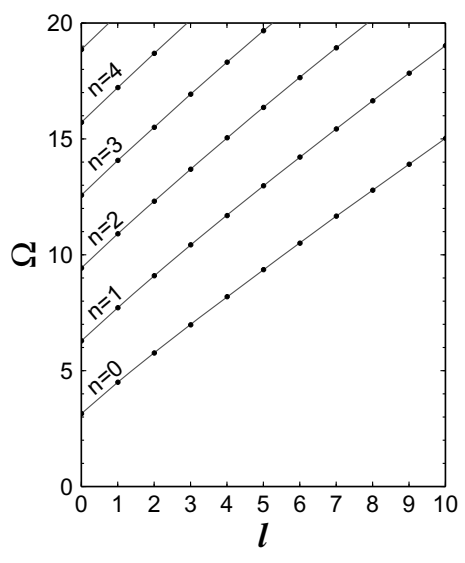

Fig. 3. The nondimensional eigenfrequencies $\Omega$ of free oscillations of a fluid sphere in vacuum are plotted versus angular order $l$. The branches are designated as the compressional modes in a fluid sphere ${ }_{n} S_{l}$, where $n$ is an integer to distinguish the branches as indicated.
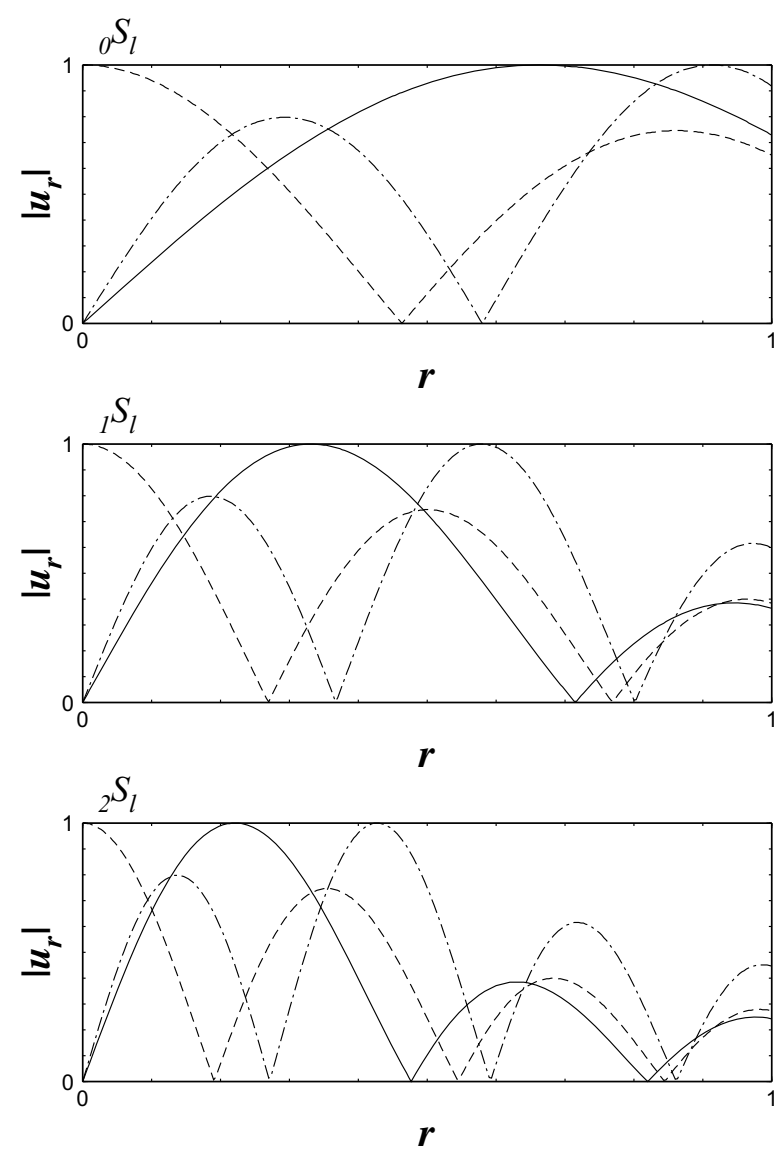

Fig. 4. The amplitude of radial displacement (the quantity in the bracket in Eq. (7)) associated with free oscillations of a fluid sphere in vacuum is shown as a function of the nondimensional distance from the center of the sphere, $r$. The eigenmodes ${ }_{0} S_{l},{ }_{1} S_{l}$ and ${ }_{2} S_{l}$ are shown for $l=0$ (solid line), $l=1$ (dashed line) and $l=2$ (dash-and-dot line). The amplitude is normalized so that the maximum in the range $0 \leq r \leq 1$ is unity. 
(a) angular frequency

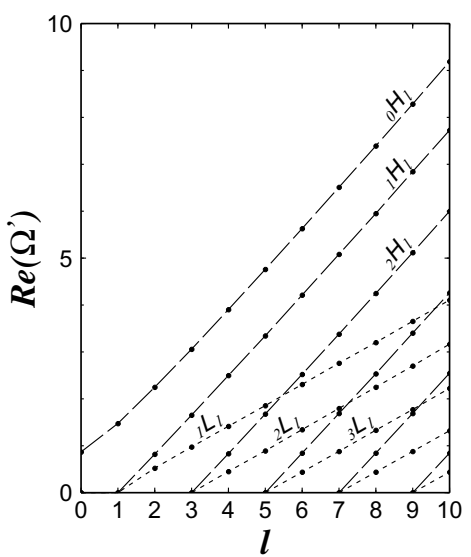

(b) attenuation coefficient

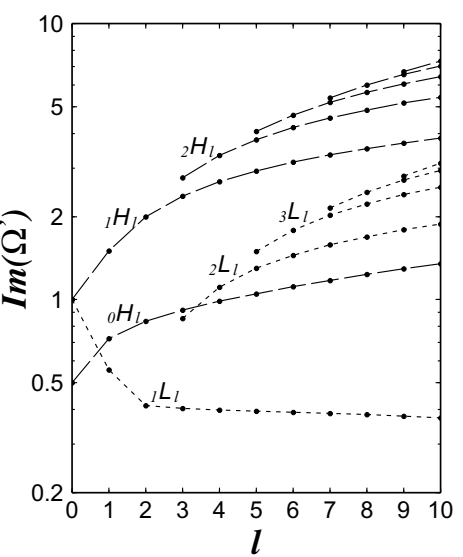

Fig. 5. The spheroidal eigenmodes of free oscillations of an infinite elastic body including a spherical void when the sound velocity ratio $\gamma$ is 0.5 . The eigenfrequency $\Omega^{\prime}$ is now a complex number; (a) the real part (angular frequency) is plotted versus angular order $l$, and (b) its imaginary part is plotted in the right which is regarded as the attenuation coefficient. The compressional modes ${ }_{n} H_{l}$ and the shear modes ${ }_{n} L_{l}$ are represented by broken and dotted lines, respectively.

(a) angular frequency

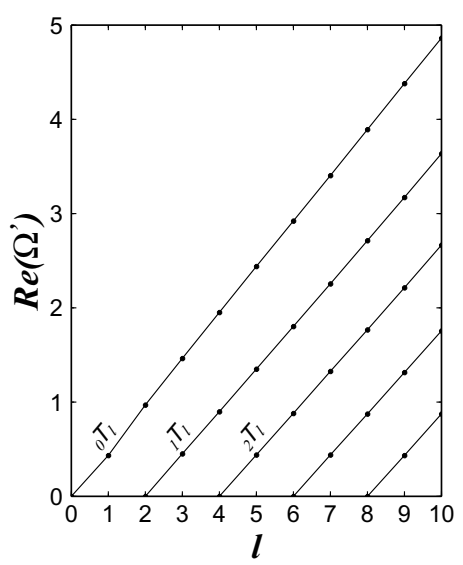

(b) attenuation coefficient

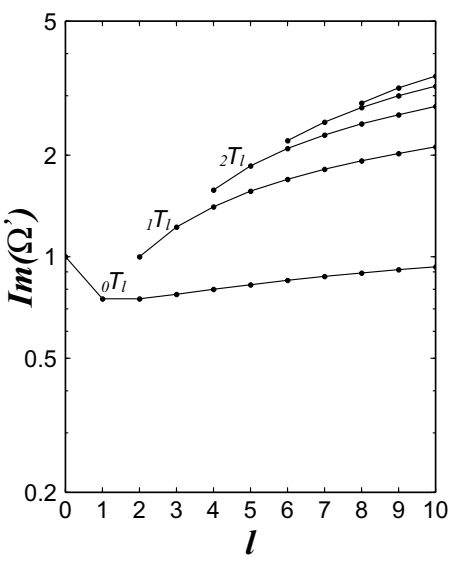

Fig. 6. The torsional eigenmodes ${ }_{n} T_{l}$ of free oscillations of an infinite elastic body including a spherical void when the sound velocity ratio $\gamma$ is 0.5 . The format is the same as that of Fig. 5 .

categorized into two groups by the difference of their slopes. The first one is a set of "compressional modes in a solid medium" of which the slope is about unity, the nondimensional $\mathrm{P}$ wave velocity in the solid medium. In contrast to the previous case, the number of branches increases along the lower right direction in Fig. 5(a). Thus, we use the symbol ${ }_{n} H_{l}(n=0,1,2, \cdots)$ to indicate the compressional modes, but now the frequency of the mode ${ }_{n} H_{l}$ is higher than that of ${ }_{n+1} H_{l}$. The other one is a set of "shear modes in a solid medium" of which the slope is $\gamma$, nondimensional S wave velocity in the solid medium. The torsional oscillations of the elastic medium, including a spherical cavity (i.e., the solutions of (24)), are shown in Fig. 6. We refer to the eigenmodes in Fig. 6 as "torsional modes in a solid medium" and use the symbol ${ }_{n} T_{l}(n=0,1,2, \cdots)$. The slope of the torsional modes is about $\gamma$.

In Fig. 5, the compressional and shear modes in the solid medium look like twin branches; that is, there is one shear mode to be paired with a compressional mode ${ }_{n} H_{l}$ and the branches of theses two modes intersect at the point $\left(l, \operatorname{Re}\left(\Omega^{\prime}\right)\right)=(2 n-1,0)$ in Fig. 5(a). We refer to the associated shear mode as the $n$th shear mode of degree $l$ and use the symbol ${ }_{n} L_{l}$. Note that ${ }_{0} L_{l}$ does not exit; there is no branch of the shear modes to be paired with ${ }_{0} H_{l}$.

In general, the attenuation coefficient $\operatorname{Im}\left(\Omega^{\prime}\right)$ of ${ }_{n} H_{l}$ is larger than that of ${ }_{n} L_{l}$, and that of the torsional mode ${ }_{n} T_{l}$ lies between them. Of particular interest is that the attenuation coefficient of the eigenmodes ${ }_{1} L_{l}$ decreases as the degree $l$ increases. These modes are generated by surface waves trapped along the spherical surface of the cavity. In this case, the surface wave is known as the Rayleigh wave but its generalization in the system described in this paper presents much interest. A detailed description on the ${ }_{1} L_{l}$ mode is given in such a general case below. 
(a) angular frequency

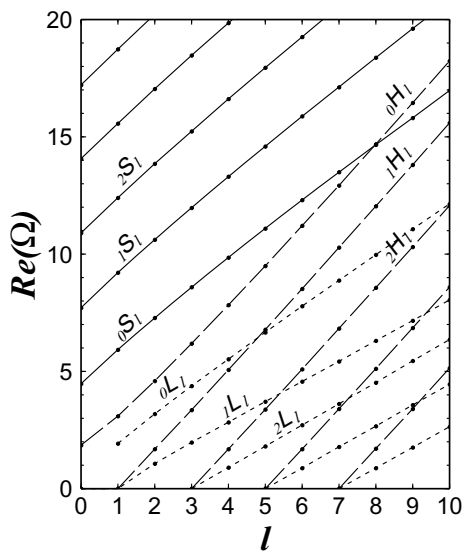

(b) attenuation coefficient

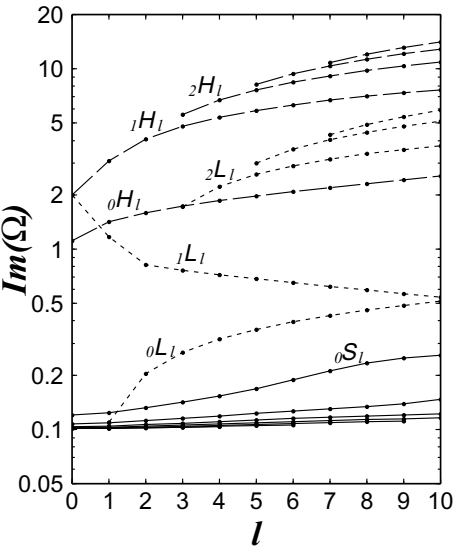

Fig. 7. The nondimensional eigenfrequencies of free oscillations of a fluid sphere embedded in an elastic medium are shown when the nondimensional parameters are $\alpha=2, \gamma=0.5$ and $\rho=5$. The real part (a) of the eigenfrequency $\Omega$ is plotted versus angular order $l$ in the left and its imaginary part (b) in the right. The compressional modes in a fluid sphere $\left({ }_{n} S_{l}\right)$, the compressional modes in a solid medium $\left({ }_{n} H_{l}\right)$ and the shear modes in a solid medium $\left({ }_{n} L_{l}\right)$ are denoted, respectively, by solid, broken and dotted lines.

\subsection{Free oscillations of a fluid sphere in an infinite elas- tic body}

The essential features of free oscillations of a fluid sphere embedded in an elastic medium can be understood as the combination of the two special cases described above. Figure 7 shows the complex nondimensional eigenfrequencies of the spheroidal oscillations for $\alpha=2, \gamma=0.5$ and $\rho=5$. At first sight, one may regard the solutions of this system as the complete sum of those of free oscillations of a fluid sphere (shown in Fig. 3) and those of free oscillations of an elastic body including a spherical cavity (shown in Fig. 5). For example, we can identify the red branches in Fig. 7 with the compressional modes in a fluid sphere ${ }_{n} S_{l}$. The difference from the previous case is that these modes have small but finite values of attenuation coefficient because the elastic waves in fluid radiate through the fluid-solid boundary into the medium outside. As shown in Fig. 8, the eigenfunctions have almost the same property as that of free oscillations of a fluid sphere.

Similarly, we can identify the black and blue branches in Fig. 7 with the compressional modes ${ }_{n} H_{l}$ and the shear modes ${ }_{n} L_{l}$, respectively. In this case, the gradient of the branches of the compressional modes is about $\alpha$ and that of the shear modes is $\alpha \gamma$, because we have taken the nondimensional $\mathrm{P}$ wave velocity in fluid as unity. The eigenmodes of the torsional oscillations are completely the same as shown in Fig. 6 because the property of the fluid sphere does not affect Eq. (24).

The most distinctive feature of this system consists in the existence of ${ }_{0} L_{l}$, which does not appear in free oscillations of a fluid sphere nor in those of a spherical cavity in an infinite elastic body. We use the symbol ${ }_{0} L_{l}$ to distinguish the new branch because this appears to be paired with ${ }_{0} H_{l}$ in Fig. 7. However, it bears characteristics common to the free oscillations of a fluid sphere. This is confirmed by the fact that the slope of the ${ }_{0} L_{l}$ branch is the same as that of the compressional modes in fluid (red branches) and that its eigenfunction has large amplitude in the fluid sphere. The
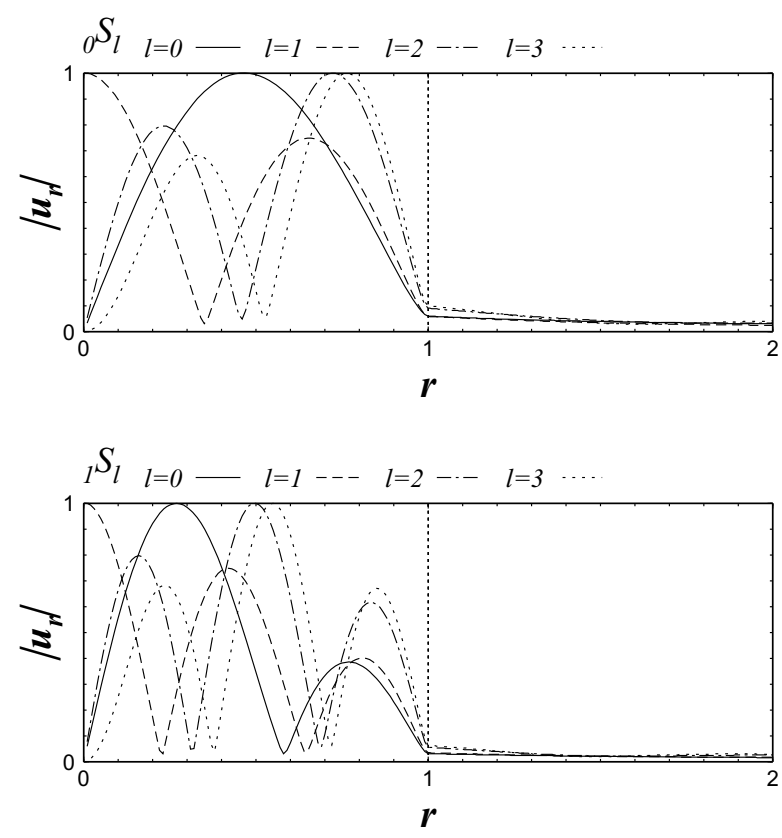

Fig. 8. Normalized amplitude of radial displacement (the quantity in the bracket in Eq. (7)) of the compressional modes in fluid ${ }_{0} S_{l}$ and ${ }_{1} S_{l}$ $(0 \leq l \leq 3)$ is shown as a function of the nondimensional distance from the center of the sphere ( $r=1$ is the fluid-solid boundary). The nondimensional parameters are the same as Fig. $7 ; \alpha=2, \gamma=0.5$ and $\rho=5$.

top panel of Fig. 9 shows the radial displacement of ${ }_{0} L_{l}$ $(1 \leq l \leq 4)$. One can readily find that these are essentially the fundamental modes of the oscillations of a fluid sphere; the fluid sphere vibrates more vigorously than the solid medium outside and there are no nodes in the range $0<r<a$. The existence of the higher-order fundamental modes which have no nodes in fluid is brought by the coupling between the fluid sphere and the outer solid medium. Although the fluid does not have shear stress, the elasticity of the outer medium sustains the deformation of the fluid- 

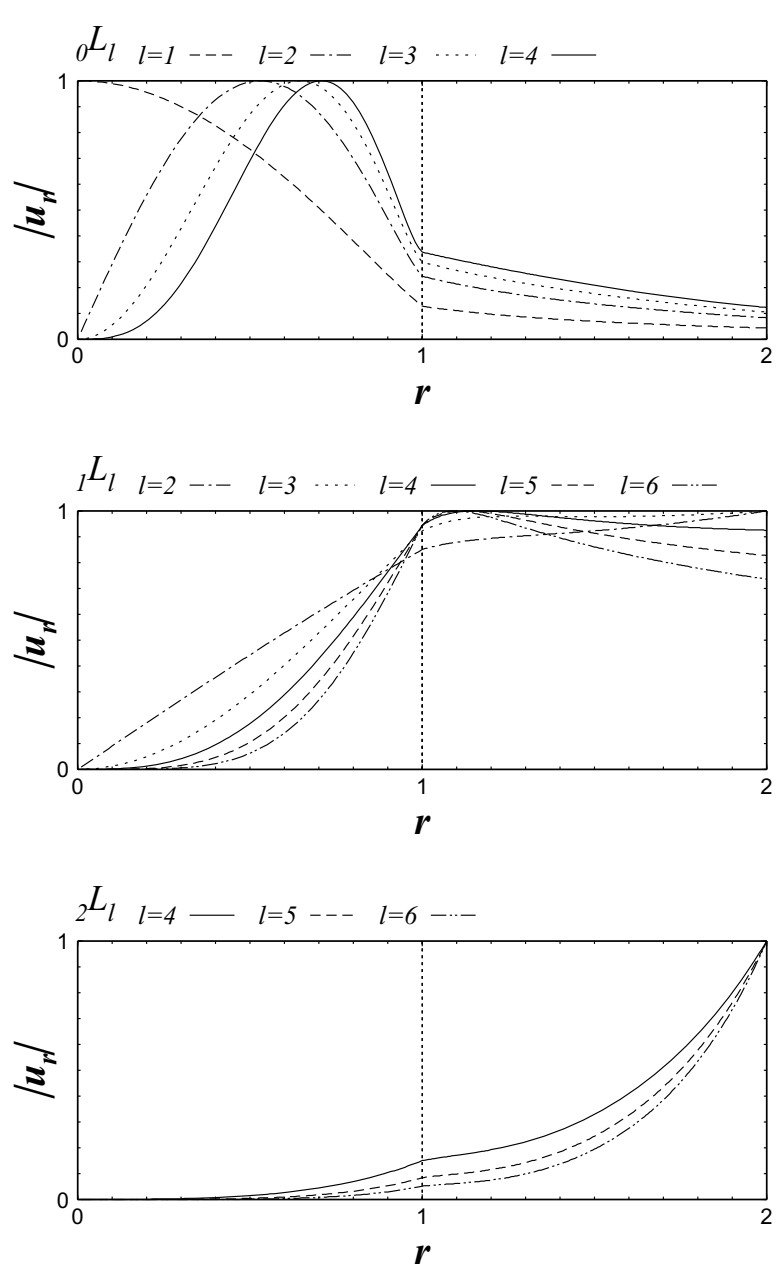

Fig. 9. Same as Fig. 8 but for the shear modes in the outer medium ${ }_{n} L_{l}(0 \leq n \leq 2)$. Note that the eigenmodes ${ }_{0} L_{l}$ are classified to the fundamental modes of a fluid sphere.

solid boundary and enables the eigenmodes ${ }_{0} L_{l}$. In particular, it is remarkable that the translation mode ${ }_{0} L_{1}$ is permitted in this system.

The middle panel of Fig. 9 shows the radial displacement of ${ }_{1} L_{l}$. As already mentioned, these modes are generated by the resonance of surface waves. In fact, one can recognize the tendency that the region where particle motion retains large amplitude gradually concentrates around the spherical boundary as the degree $l$ increases. The surface wave which is trapped around the fluid-solid boundary is called the Stoneley wave (Aki and Richards, 1980). Thus, it is appropriate that these modes are designated as "Stoneley modes". The Stoneley modes are also distinguished by the fact that the attenuation coefficient decreases to zero as the angular order increases, as seen in the right-hand side of Fig. 7.

Other eigenmodes ${ }_{n} L_{l}$ of $n \geq 2$ have the properties as the shear modes in the outer solid medium. For example, the radial displacement of ${ }_{2} L_{l}$ in the solid medium, shown in the bottom panel of Fig. 9, is significantly larger than that in the fluid. Similarly, the radial displacement of the compressional modes ${ }_{n} H_{l}$ is amplified in the region outside the sphere, as seen in Fig. 10. Only the mode ${ }_{0} H_{l}$ has different features from others; it retains relatively large amplitude
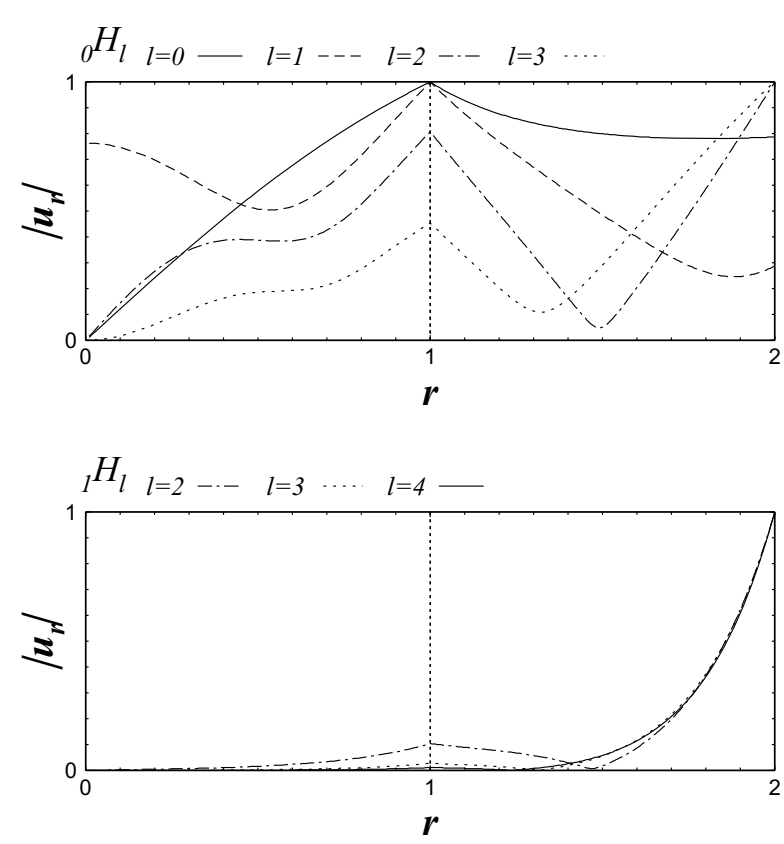

Fig. 10. Same as Fig. 8 but for the compressional modes in the outer medium $_{n} H_{l}(n=0,1)$.

in the fluid sphere and around the fluid-solid boundary, especially in a lower-order mode. This is because the branch of ${ }_{0} H_{l}$ weakly interacts with the branches of ${ }_{0} L_{l}$ and ${ }_{1} L_{l}$. However, the higher-order modes of ${ }_{0} H_{l}$ lies in the category of the compressional modes in a solid medium.

Of further interest is the parameter effects on the eigenmodes of the system. Though there are three independent nondimensional parameters $\alpha, \gamma$ and $\rho$, we restrict our interest to the effects of $\alpha$ and $\rho$. In most cases, the ratio of the $\mathrm{S}$ and $\mathrm{P}$ wave velocities $\gamma$ in the uppermost crust lies between 0.4 and 0.6 , and it contributes only a minor effect in this range. Figure 11 shows the angular frequency of several eigenmodes as a function of $\alpha$ for some values of $\rho$. The nondimensional parameter $\gamma$ is fixed to 0.5 . We can find essentials of the parameter effects in Fig. 11. First, the angular frequency of the compressional mode in a fluid sphere, ${ }_{n} S_{l}$ and ${ }_{0} L_{l}$, is approximately constant against the change of the nondimensional parameters. However, it makes only a slight difference of the constant value whether $\alpha \rho<1$ or $\alpha \rho>1$. When $\alpha \rho<1$, the situation is like the free oscillations of a fluid sphere in vacuum; the boundary condition can be approximated by the stress-free condition. When $\alpha \rho>1$, on the other hand, the situation is like the free oscillations of a fluid sphere in a rigid body; the boundary condition is $u_{r}=0$ at $r=a$. In this respect, the attenuation coefficient is larger when $\alpha \rho \simeq 1$, and it decreases to zero as the impedance contrast $\alpha \rho$ becomes much greater or much smaller than unity.

Second, the compressional mode in a fluid sphere ${ }_{0} L_{l}$ $(l \geq 1)$ which has no nodes in the range $0<r<a$ does not always exist but disappears when $\alpha \rho<1$. This is because the nature of the vibrations when $\alpha \rho<1$ is essentially the same as that of free oscillations of a fluid sphere in vacuum, in which the elasticity of the outer medium has less influence on the deformation of the fluid sphere. Similarly, the angular 
$\operatorname{Re}(\Omega)$
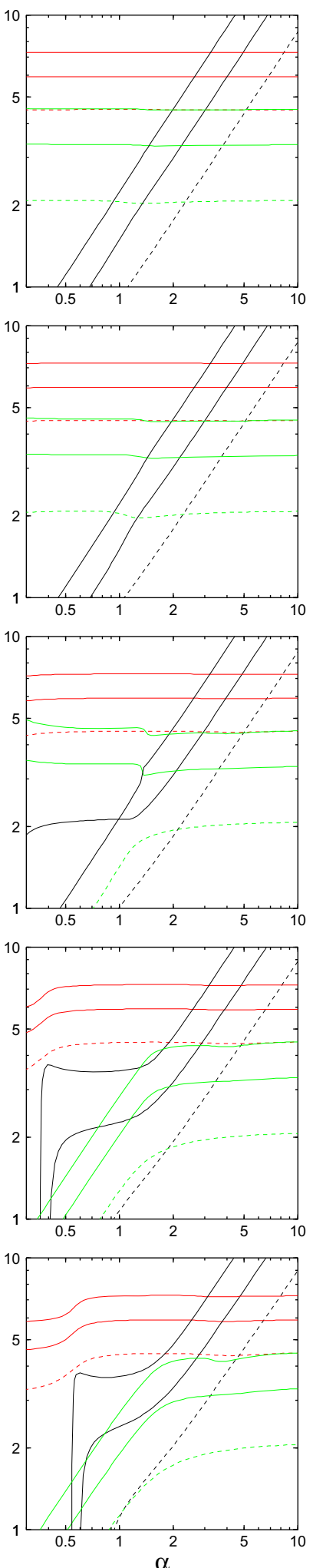

$\operatorname{Im}(\Omega)$
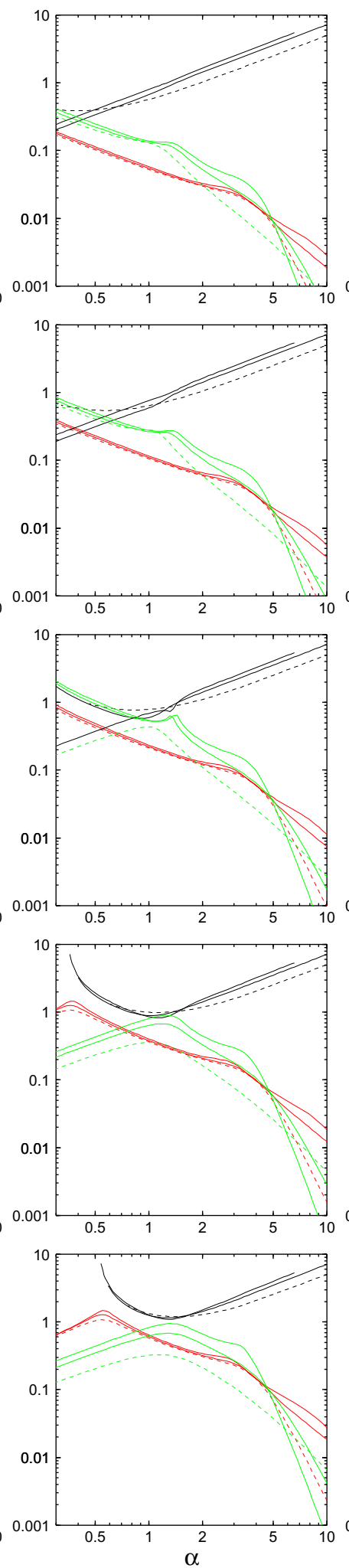

$\zeta=\operatorname{Im}(\Omega) / \operatorname{Re}(\Omega)$

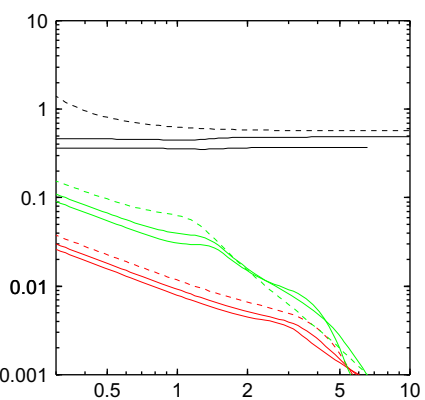

$\rho=20.0$
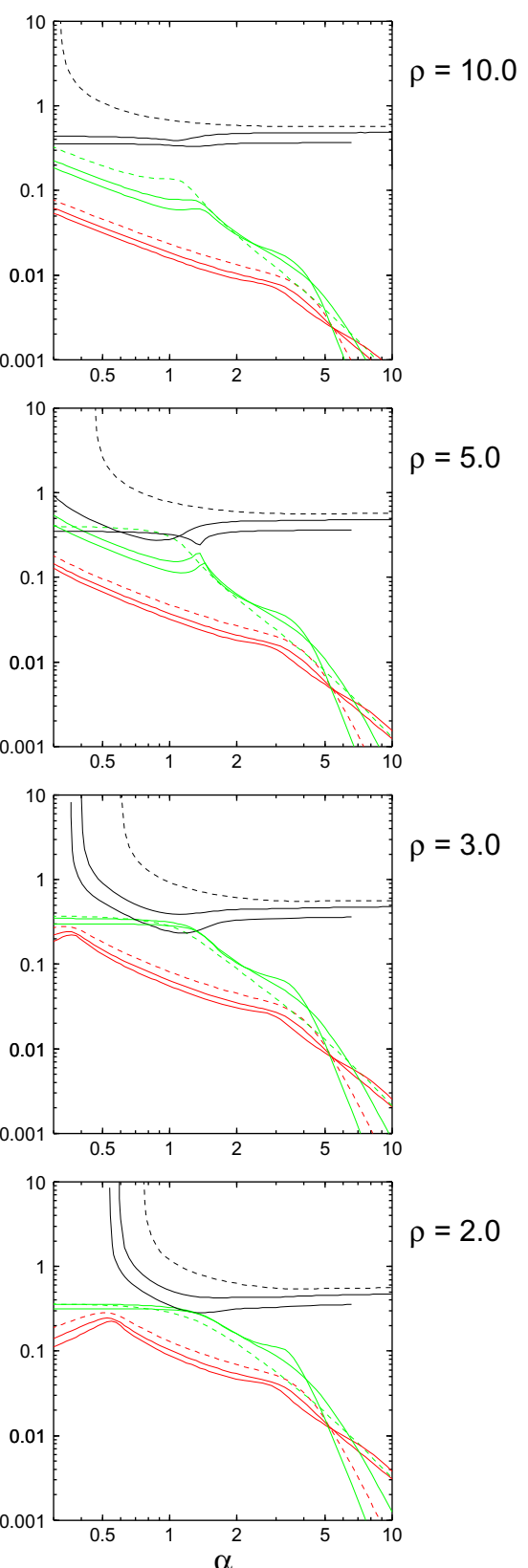

Fig. 11. Complex eigenfrequencies of ${ }_{0} S_{l}$ (red lines; $0 \leq l \leq 2$ ), ${ }_{0} L_{l}$ (green lines; $1 \leq l \leq 3$ ) and ${ }_{0} H_{l}$ (black lines; $0 \leq l \leq 2$ ) are shown as functions of $\alpha$ for, from top to bottom, $\rho=20,10,5,3$ and 2. The nondimensional parameter $\gamma$ is 0.5 . Left, middle and right panels are, respectively, angular frequency, attenuation coefficient, and the ratio of the two. ${ }_{0} S_{0},{ }_{0} L_{1}$ and ${ }_{0} H_{0}$ are shown as broken lines. 
Table 1. Frequency and its attenuation coefficient of spectral peaks of the long-period event observed at four sites (KAC, KAH, KAM and KAN). There are 16 peaks detected (See Figs. 13 and 14). The mean value and rms of the attenuation coefficients are shown in the right column.

\begin{tabular}{cllllc}
\hline frequency, $f[\mathrm{~Hz}]$ & \multicolumn{5}{c}{ attenuation coefficient, $\epsilon[\mathrm{Hz}]$} \\
\cline { 2 - 6 } & KAC & KAH & KAM & KAN & mean \\
\hline 1.73 & 0.0315 & 0.0339 & 0.0309 & 0.0310 & $0.0318 \pm 0.0012$ \\
3.19 & 0.180 & 0.159 & 0.0976 & 0.137 & $0.144 \pm 0.030$ \\
3.65 & 0.0877 & 0.0829 & 0.0864 & 0.0734 & $0.0826 \pm 0.0056$ \\
5.79 & 0.0665 & 0.0717 & 0.0796 & 0.0762 & $0.0735 \pm 0.0050$ \\
6.65 & 0.0905 & 0.0861 & 0.0844 & 0.152 & $0.103 \pm 0.028$ \\
7.14 & 0.166 & 0.131 & 0.172 & 0.121 & $0.148 \pm 0.022$ \\
7.45 & 0.134 & 0.103 & 0.126 & 0.146 & $0.127 \pm 0.016$ \\
7.92 & 0.0965 & 0.0924 & 0.108 & 0.0618 & $0.0897 \pm 0.0171$ \\
8.11 & 0.0747 & 0.0705 & 0.0862 & 0.0855 & $0.0792 \pm 0.0068$ \\
8.77 & 0.115 & 0.131 & 0.0963 & 0.105 & $0.112 \pm 0.013$ \\
10.17 & 0.0877 & 0.0691 & 0.0749 & 0.0358 & $0.0669 \pm 0.0192$ \\
10.60 & 0.114 & 0.122 & 0.106 & 0.146 & $0.122 \pm 0.015$ \\
11.43 & 0.167 & 0.112 & 0.129 & 0.0917 & $0.125 \pm 0.028$ \\
11.81 & 0.126 & 0.179 & 0.109 & 0.0823 & $0.124 \pm 0.035$ \\
12.27 & 0.125 & 0.0826 & 0.138 & 0.106 & $0.113 \pm 0.021$ \\
13.61 & 0.0795 & 0.103 & 0.107 & 0.0939 & $0.0959 \pm 0.0105$ \\
\hline
\end{tabular}

frequency of some compressional modes in a solid medium, say ${ }_{0} H_{0}$, becomes to zero when $\alpha \rho<1$. Once the frequency falls into zero, the solution becomes two pure imaginaries (Fujita et al., 1995). Around the critical point where ${ }_{0} L_{l}$ disappears, this branch strongly interacts with the branch ${ }_{0} H_{l}$ and it is difficult to interpret which branch plays a role as the fundamental compressional mode in fluid.

Third, the complex eigenfrequencies of the compressional and shear modes in a solid medium are proportional to $\alpha$. Thus, the ratio of the attenuation coefficient to the angular frequency $\zeta=\operatorname{Im}(\Omega) / \operatorname{Re}(\Omega)$ is almost constant against the change of $\alpha$. It should be noticed that the value of $\zeta$ is at least larger than 0.1 for any ${ }_{n} H_{l}$ and ${ }_{n} L_{l}$ of $n \geq 1$ as seen in the right panels of Fig. 11. For example, the amplitude of the vibrations is damped down to $1 / e$ in each cycle of the vibration if $\zeta=1 / 2 \pi \simeq 0.159$. Therefore, we can recognize that the compressional and shear modes in solid are more radiative than this situation. Though the detail is not shown, the torsional modes are the same as the compressional and shear modes in a solid medium in nature; its angular frequency is proportional to $\alpha$, and $\zeta$ is at least larger than 0.1 .

\section{Application to the Long-Period Volcanic Earth- quake}

The seismograms of the long-period volcanic earthquake to which we will apply the model described in the previous sections have been shown in Fig. 1. The earthquake occurred beneath the summit crater of Asama volcano, Japan. In Fig. 1, the data obtained at the nearest four observation points (KAC, KAH, KAM and KAN) to the hypocenter are plotted. The location of these observation points is shown in Fig. 12. From a precise seismic study (Tsuji et al., 1998),

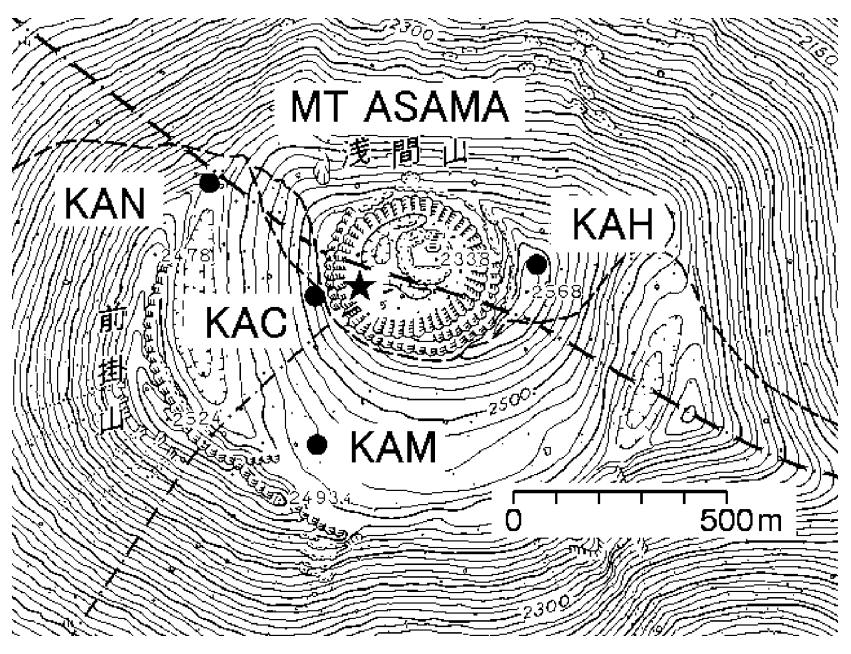

Fig. 12. The topographic map around the summit of the Asama volcano. The interval of thin contours is $10 \mathrm{~m}$. Four seismometers (KAC, KAH, KAM and KAN) are installed around the summit crater and shown as dots. The hypocenter of the observed long-period event is shown as a star which is at a depth of $800 \mathrm{~m}$ below KAC.

it is known that the hypocenter is about $800 \mathrm{~m}$ deep beneath KAC, as shown in Fig. 12. Although the seismometers recorded three components of ground vibrations, we only use the vertical motion data because other components seems to have considerable noises.

Figure 13 shows the spectra obtained by Fourier transforming the data in the range $5.9 \mathrm{~s}<t<74.1 \mathrm{~s}$ in Fig. 1. The primary peak is the one of which the characteristic frequency is about $1.73 \mathrm{~Hz}$. The amplitude of the other peaks are several percent of that of the primary one. We detected 

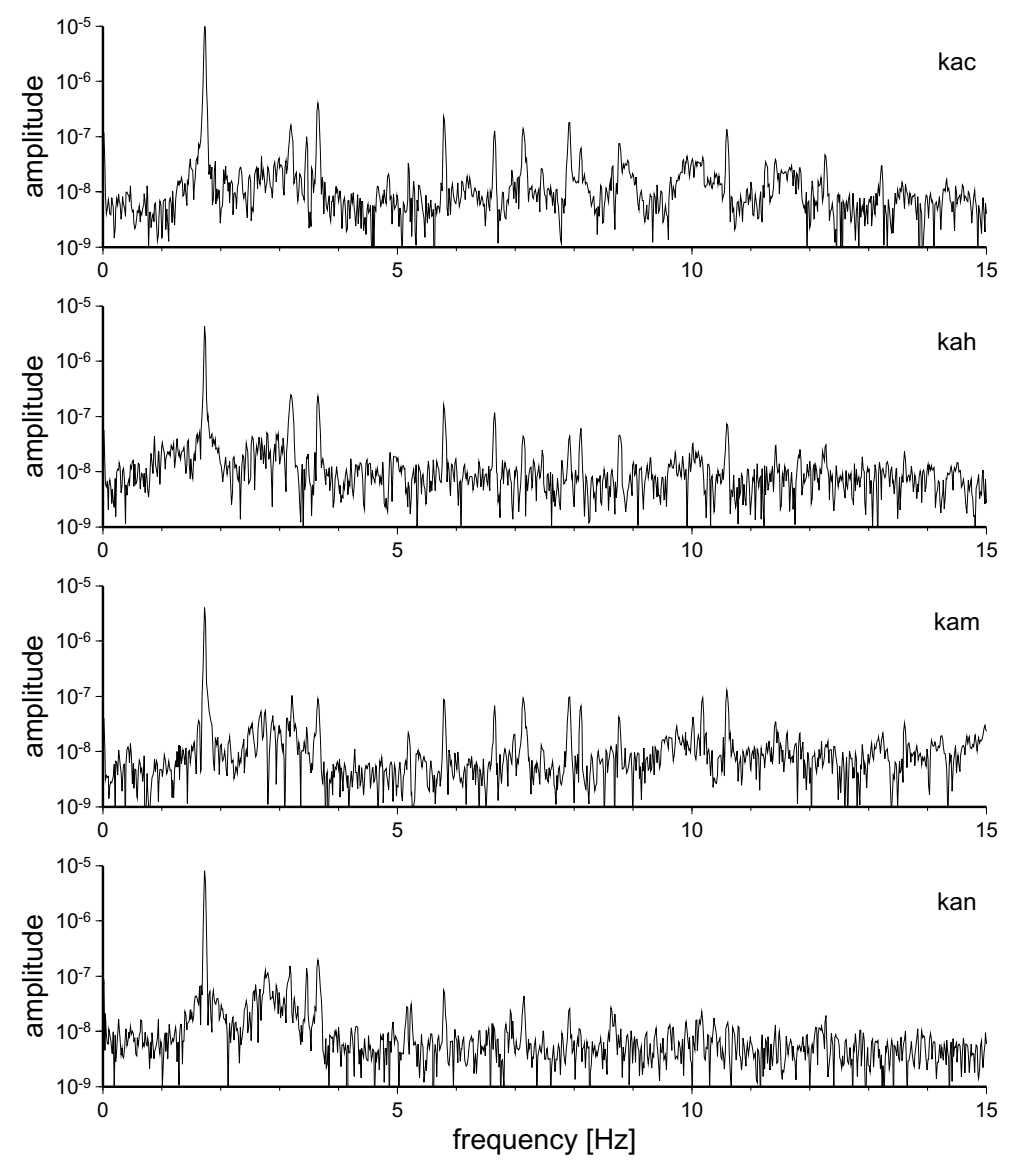

Fig. 13. The amplitude spectra of the data shown in Fig. 1 of which the time domain $5.9 \mathrm{~s}<t<74.1 \mathrm{~s}$ is used in the calculation.

16 characteristic peaks including the main peak in the range $f<15 \mathrm{~Hz}$ (Table 1). It appears that the amplitudes of the primary oscillations $(f=1.73 \mathrm{~Hz})$ are consistent with the distance from the hypocenter.

The attenuation coefficient of the characteristic oscillations can be obtained by Fourier transforming the data with many staggered windows and estimating the time variation of the peak amplitude. Figure 14 shows the time variation of the amplitude of various peaks. From the slope of the lines in Fig. 14, we can estimate the attenuation coefficient $\epsilon[\mathrm{Hz}]$ defined in such a way that the amplitude is proportional to $e^{-\epsilon t}$. For example, the attenuation coefficient of the primary peak is $0.032 \pm 0.001 \mathrm{~Hz}$. The attenuation coefficients of the other peaks are also estimated but with less precision (Table 1).

We consider that the primary peak $(f=1.73 \mathrm{~Hz}$ and $\epsilon=0.032 \mathrm{~Hz}$ ) is the compressional mode in a fluid sphere of the lowest frequency, because there are no notable peaks before it and its attenuation coefficient is very small. In general, the lowest frequency belongs to ${ }_{0} L_{1}$ mode, a simple translation mode of a fluid sphere in an elastic medium.

To estimate the model parameters $\alpha, \gamma$ and $\rho$, we direct attention to a quantity $\zeta=\operatorname{Im}(\Omega) / \operatorname{Re}(\Omega)$. This quantity is related to the observables $f$ and $\epsilon$ as

$$
\zeta=\frac{\epsilon}{2 \pi f}
$$

In this case, we have $\zeta=(2.95 \pm 0.09) \times 10^{-3}$ for the pri- mary peak from observation. If we assume that the primary peak corresponds to ${ }_{0} L_{1}$ mode, the value of $\zeta$ of ${ }_{0} L_{1}$ mode, which is a function of $\alpha, \gamma$ and $\rho$, satisfies

$$
\zeta\left({ }_{0} L_{1}\right)=\zeta\left({ }_{0} L_{1} ; \alpha, \gamma, \rho\right) \simeq 2.95 \times 10^{-3} .
$$

The relation between $\alpha$ and $\rho$ is shown in Fig. 15 on condition that Eq. (29) is satisfied for $\gamma=0.5$. Under this condition, the nondimensional frequencies of several compressional modes in a fluid sphere which have small values of $\zeta$ are also shown in Figs. 16 and 17. One can see that the real part of the eigenfrequency is almost constant, even though the nondimensional parameters vary. Thus, we cannot determine the nondimensional parameters that best fit the data from the information of frequency. However, the imaginary part sensitively depends on the nondimensional parameters, as shown in the bottom panels of Figs. 16 and 17, giving the possibility of estimating the model parameters.

Figure 18 shows the extent to which the observed spectral peaks are explained by the model. We obtained a relation between the observed frequency and the calculated angular frequency by fitting a line to the intersections of vertical $(\operatorname{Re}(\Omega))$ and horizontal $(f[\mathrm{~Hz}])$ lines in the diagram shown. If the relation is written in the form

$$
f=b \operatorname{Re}(\Omega)
$$

our preferred model indicates $b=\alpha_{1} / 2 \pi a=0.933 \mathrm{~Hz}$. It can be said that its proportionality is reasonably good; all 


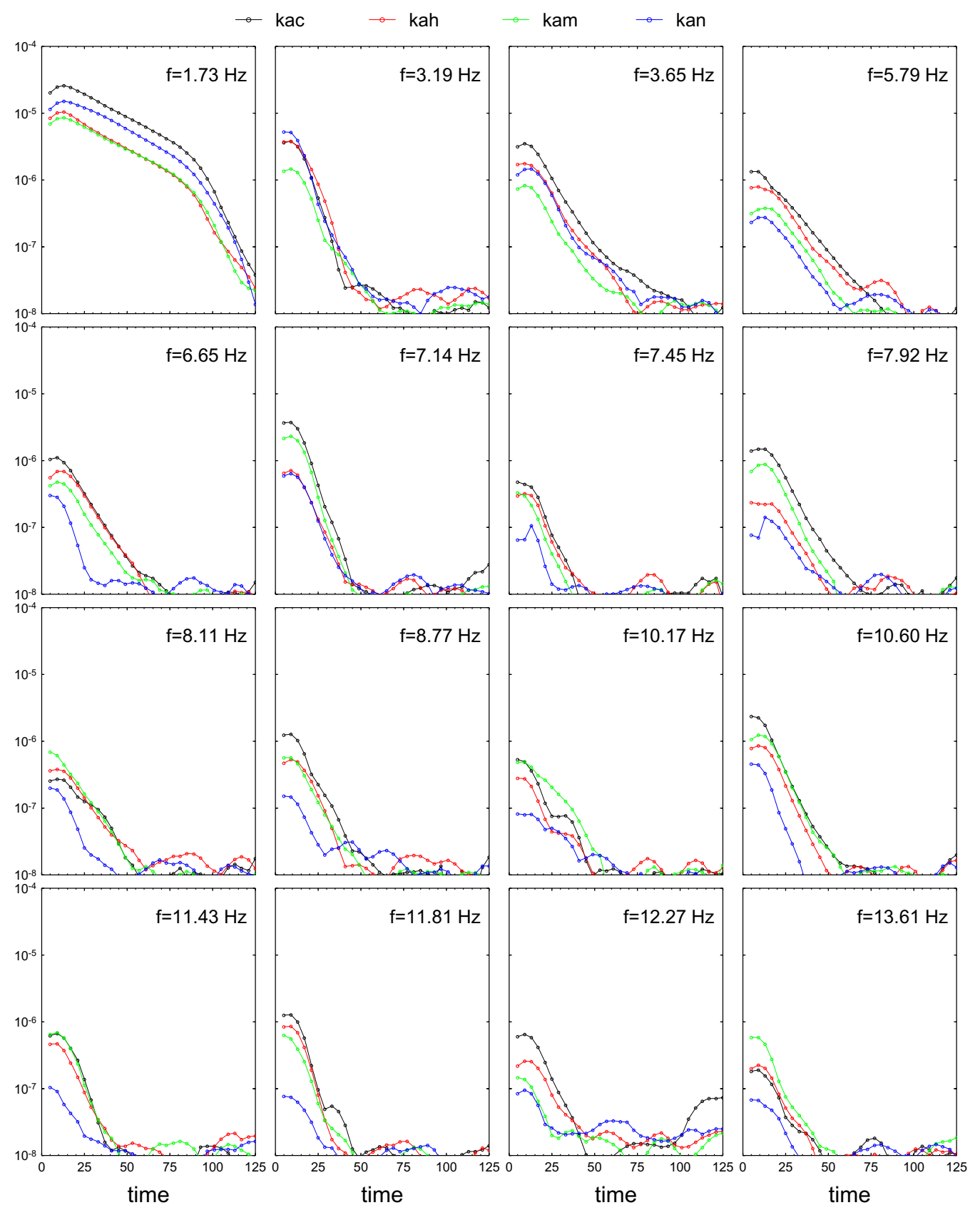

Fig. 14. Amplitude of the spectral peak $f$ is shown as a function of time. Each dot at time $t$ is obtained by Fourier transforming the data with the data window $(t \pm 17) \mathrm{s}$. The frequencies observed at KAC, KAH, KAM and KAN are shown as black, red, green, and blue lines, respectively.

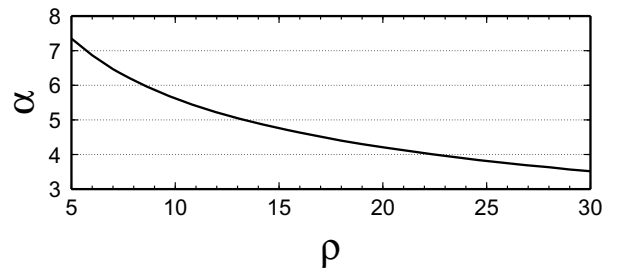

Fig. 15. The relation between $\alpha$ and $\rho$ on condition that the ratio of the imaginary to real parts of the eigenfrequency $\Omega$ of ${ }_{0} L_{1}$ is fixed to $2.95 \times 10^{-3}$ for $\gamma=0.5$ (i.e., the condition that Eq. (29) is satisfied). the peaks are explained with an error of less than $\sim 3 \%$ but with two exceptions, $f=3.65 \mathrm{~Hz}$ and $f=1.73 \mathrm{~Hz}$. The problem is that there exist a few eigenmodes which are not excited to vibrations as shown in Fig. 18. There are some situations in which an eigenmode does not reveal itself. We can imagine that the source function of the long-period volcanic event does not include the eigenfunction of that mode; but this is rather self-seeking. Instead, it may be reasonable that the eigenmode could not be observed because its attenuation coefficient was so large. Re-examining Figs. 16 and 17 from this point of view, we find that some of the eigenmodes have relatively large values of $\operatorname{Im}(\Omega)$ at a certain parameter. Indeed, the nondimensional attenuation coefficients of ${ }_{0} L_{3}$, ${ }_{0} L_{4}$ and ${ }_{0} L_{5}$ are larger than the others when $\rho \sim 25$ and, 

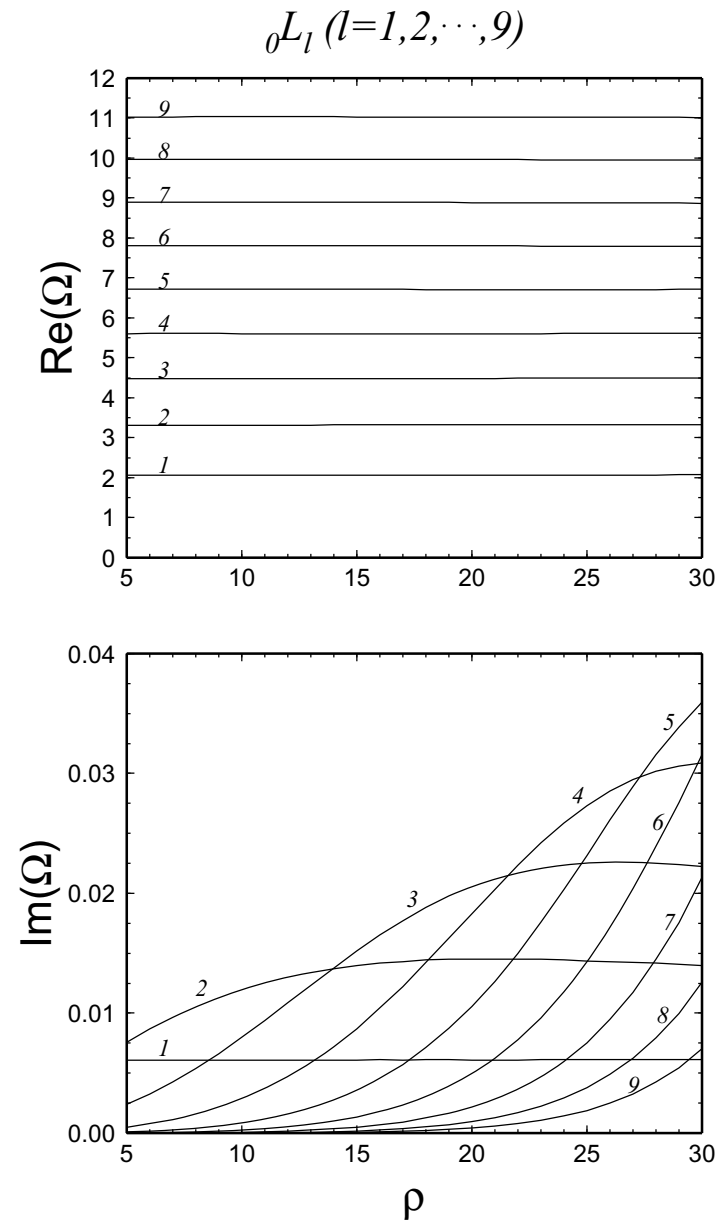

Fig. 16. The real and imaginary parts of the eigenfrequencies of ${ }_{0} L_{l}$ $(1 \leq l \leq 9)$ are plotted as functions of $\rho$. The other nondimensional parameters ( $\alpha$ and $\gamma$ ) are determined by Fig. 15; $\alpha$ is a function of $\rho$ and $\gamma=0.5$. A number to a line denotes the degree $l$ of the eigenmode.

at the same time, the corresponding peaks to these eigenmodes are absent, as seen in Fig. 18. In this case, we adopt the criterion that the eigenmode of which the imaginary part of its nondimensional frequency is larger than 0.0175 cannot be observed due to its rapid damping. Then we have a constraint $\rho>23$ because $\operatorname{Im}(\Omega)$ of ${ }_{0} L_{3},{ }_{0} L_{4}$ and ${ }_{0} L_{5}$ are all greater than 0.0175 (see Fig. 16). And further, $\operatorname{Im}(\Omega)$ of ${ }_{0} L_{6}$ should be less than 0.0175 because this eigenmode seems to be excited into resonance, according to Fig. 18. Therefore we can impose another condition $\rho<26$ (see Fig. 16). Taking account of the error in estimating the attenuation coefficients from observation, we can determine the nondimensional parameters in the range $\rho \simeq 24 \pm 2$ and, correspondingly, $\alpha \simeq 3.9 \mp 0.2$. Figures 18 and 19 indicate our preferred model in which $\alpha=3.88, \gamma=0.5$ and $\rho=24$. The absence of ${ }_{0} L_{3},{ }_{0} L_{4},{ }_{0} L_{5}$ and the higher-order modes of ${ }_{0} S_{l}(l \geq 4)$ is well explained by the high attenuation of the calculated eigenmodes. The result is summarized in detail in Table 2.

\section{Summary and Discussion}

We described theoretical and applicable aspects of free oscillations of a fluid sphere in an infinite elastic medium.
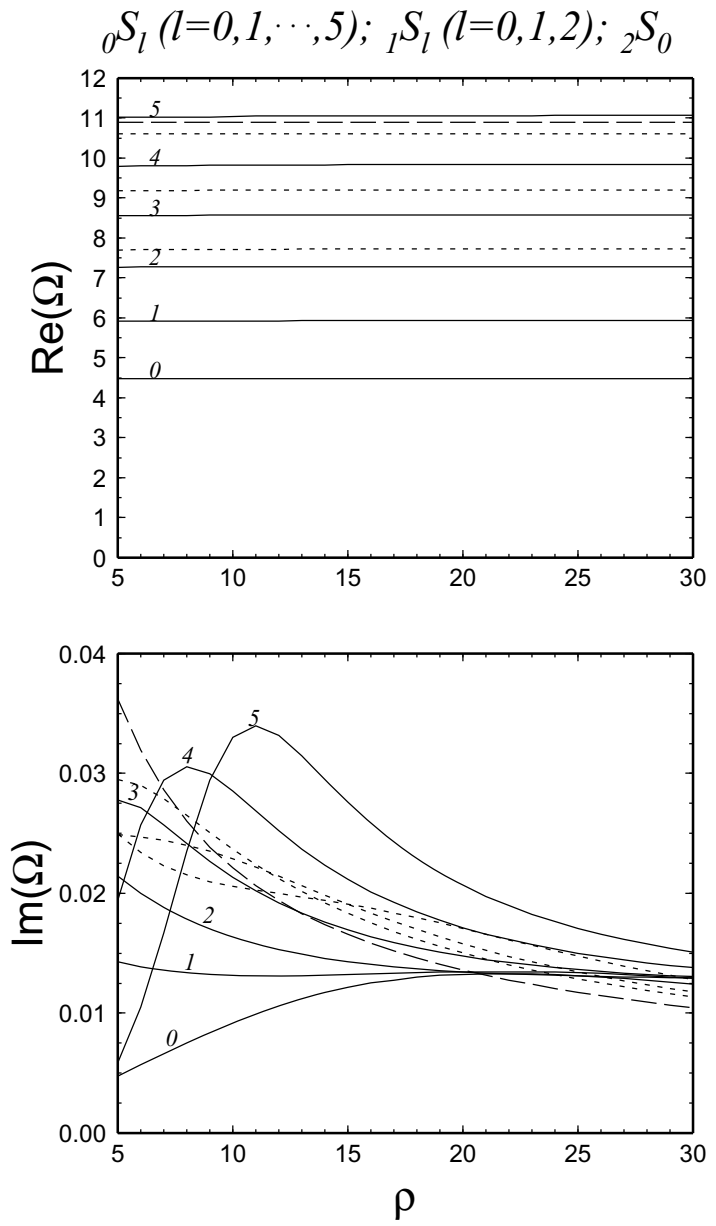

Fig. 17. Same as Fig. 16, but for ${ }_{0} S_{l}(0 \leq l \leq 5),{ }_{1} S_{l}(0 \leq l \leq 2)$ and ${ }_{2} S_{0}$. A number to a line denotes the degree of the eigenmode ${ }_{0} \bar{S}_{l}$.

In the first half of this paper, the eigenequation of threedimensional free oscillations of the system is derived making use of the spherical harmonic and spherical Bessel expansions. The solutions are categorized into five different types of eigenmodes: (1) compressional modes in fluid, ${ }_{n} S_{l}$ and ${ }_{0} L_{l} ;(2)$ compressional modes in a solid medium, ${ }_{n} H_{l}$; (3) shear modes in a solid medium, ${ }_{n} L_{l}(n \geq 2)$; (4) Stoneley modes, ${ }_{1} L_{l}$; and (5) torsional modes, ${ }_{n} T_{l}$. In these modes, the compressional modes in fluid, originating from free oscillations of a fluid sphere in vacuum, is the most important to be applied to long-period volcanic earthquakes because they have small attenuation coefficients. In particular, the quantity $\zeta=\operatorname{Im}(\Omega) / \operatorname{Re}(\Omega)$ of the compressional modes in fluid is smaller than $\mathrm{O}\left(10^{-1}\right)$, while that of the other modes caused by the vibrations of the outer medium is much larger. Therefore, it is enough for us to take account of the modes ${ }_{n} S_{l}$ and ${ }_{0} L_{l}$ to apply the model to a long-coda volcanic earthquake.

The eigenfrequency obtained from (25) or (27) is a complex number; its real part is the angular frequency and imaginary part is the attenuation coefficient, since the amplitude of the displacement is written as $u \propto \exp [i \operatorname{Re}(\Omega) t-$ $\operatorname{Im}(\Omega) t]$, where $\operatorname{Im}(\Omega)>0$. At the same time, the displacement at the point very far from the center of the fluid sphere can be written as $u \propto \exp [-i \operatorname{Re}(\Omega) r+\operatorname{Im}(\Omega) r]$, 


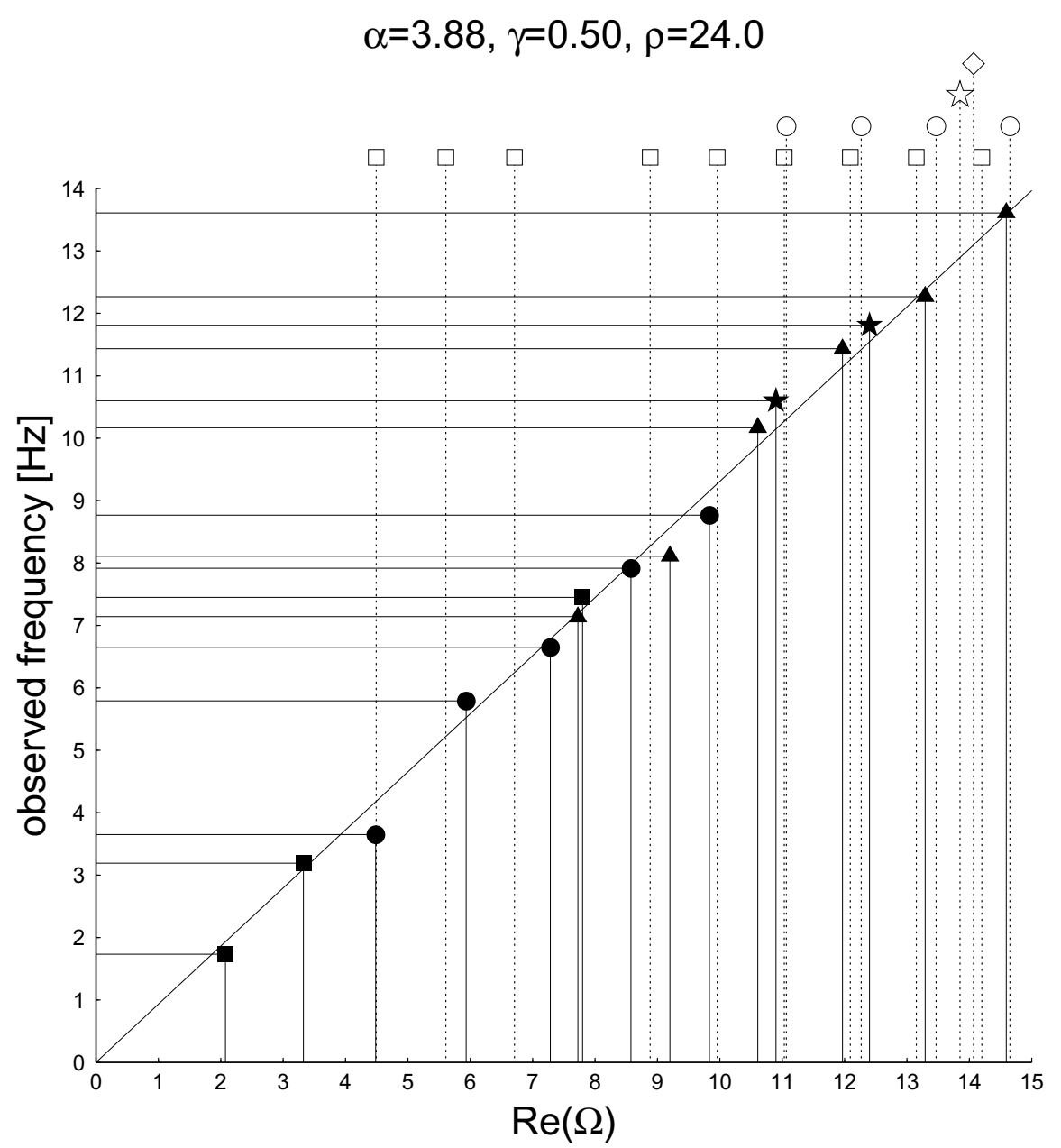

Fig. 18. The observed characteristic frequencies $f[\mathrm{~Hz}]$ are represented by horizontal lines, while the nondimensional frequencies $\operatorname{Re}(\Omega)$ calculated for $\alpha=3.88, \gamma=0.5$ and $\rho=24$ are represented by vertical lines. The intersections of the horizontal and vertical lines are represented by a filled symbol; the square, circle, triangle and star denote that the observed frequencies are explained by ${ }_{0} L_{l},{ }_{0} S_{l},{ }_{1} S_{l}$ and ${ }_{2} S_{l}$, respectively. The vertical dotted line denotes that the calculated eigenmode is not observed; the absent eigenmode is classified by using the same symbol but colored white. The diamond denotes the eigenmode ${ }_{3} S_{l}$.

because the spherical Hankel function of the second kind of degree $l$ takes the form $h_{l}^{(2)}(z) \sim i^{l+1} z^{-1} \exp (-i z)$ at infinity $(|z| \rightarrow \infty)$, where $z \propto \Omega r$ is a complex variable. Therefore, the eigenfunction is inevitably forced to diverge in the region far from the fluid sphere. Figures 9 and 10 show the tendency that the compressional and shear modes in a solid medium are more affected by this divergent problem, because they have large attenuation coefficients. This problem is due to the adoption of the radiative boundary condition at infinity which permits only the outgoing elastic waves there. However, our practical interest is directed to the compressional modes in fluid of which the attenuation coefficient is small enough $(\zeta<0.1$ at least). We consider that this problem can be avoided if we are concerned about ${ }_{n} S_{l}$ and ${ }_{0} L_{l}$ in the application.

Normal modes of a spherically symmetric solid sphere in vacuum are orthogonal to each other and there is no interaction among them. In this model, however, the eigenfunctions are not orthogonal to each other due to the radiative boundary condition at infinity. And further, they interact with each other to some extent. For example, the eigenfunctions of the lower-order modes of ${ }_{0} H_{l}$ retain large amplitude in the fluid sphere as well as in the solid medium outside (Fig. 10), indicating that these modes have the characteristic common to the compressional modes in fluid. We also find that an eigenmode significantly influences another eigenmode and there occurs a strong interaction between them when the complex eigenfrequencies are close to one another (Fig. 11). These features are intrinsic to the present situation where the resonance ensues in an infinite elastic body. Therefore, we must take care of the difference from the free oscillations of a solid (or fluid) sphere in vacuum which has been well studied.

In this study, we found a new type of eigenmode, ${ }_{0} L_{l}$ $(l \geq 1)$, generated by the interactions between fluid and solid media. These modes are categorized as one of compressional modes in fluid and have small $\zeta$ values. Their eigenfunctions have no nodes in the sphere except at its center and the boundary and they are maintained by finite elasticity outside the fluid sphere. If the cavity is filled with a solid, this type of vibration has no interest but is a wellknown branch of free oscillations of an elastic sphere. In the present situation, however, the fluid-solid interaction sustains deformation of boundary shape and even enables a 


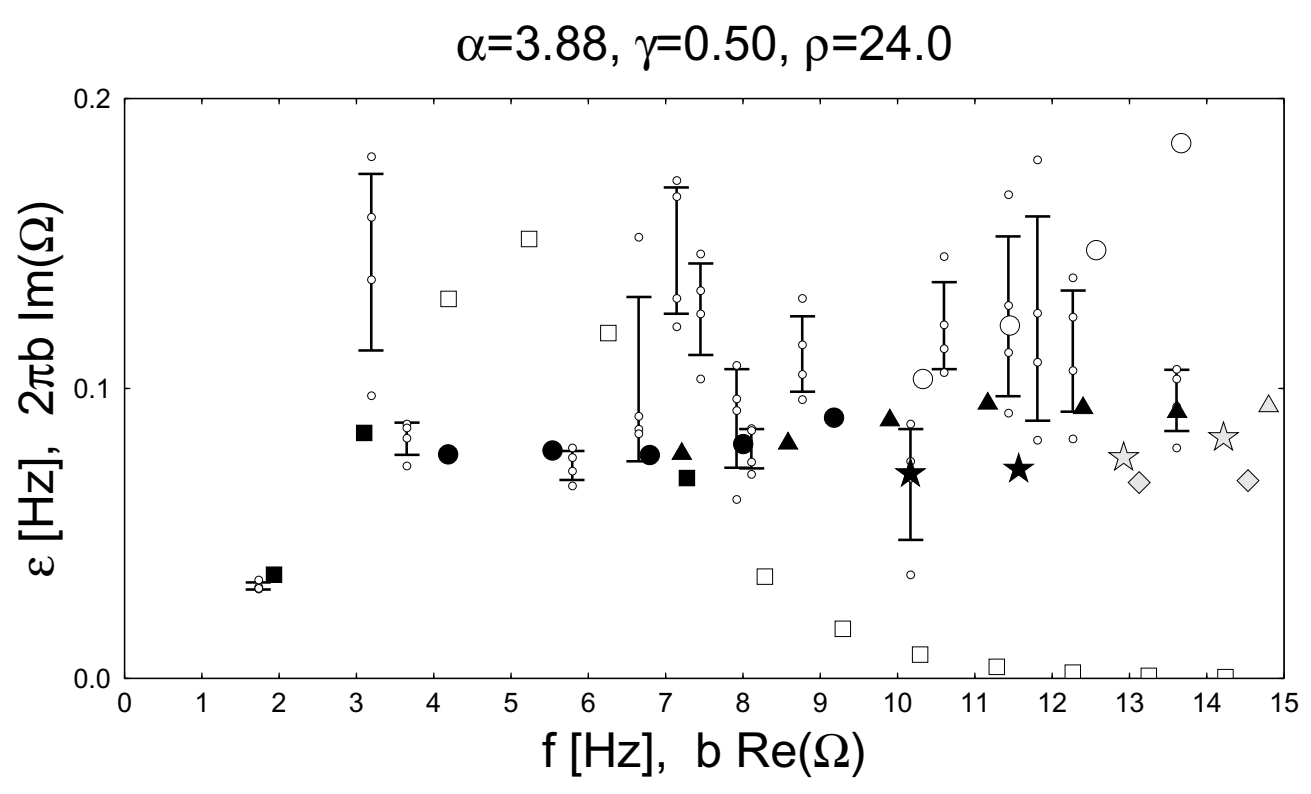

Fig. 19. The observed characteristic frequencies are plotted in the $f-\epsilon$ plane as small dots. For each $f$ [Hz], a standard deviation from the mean value of $\epsilon[\mathrm{Hz}]$ for four observation points is represented by a vertical bar. The eigenfrequencies calculated for $\alpha=3.88, \gamma=0.5$ and $\rho=24$ are also plotted in the same plane. In order to dimensionalize them, the relations $f=b \cdot \operatorname{Re}(\Omega)$ and $\epsilon=2 \pi b \cdot \operatorname{Im}(\Omega)$ are adopted, where $b=0.933 \mathrm{~Hz}$. The eigenfrequencies of ${ }_{0} L_{l}(1 \leq l \leq 13),{ }_{0} S_{l}(0 \leq l \leq 8),{ }_{1} S_{l}(0 \leq l \leq 6),{ }_{2} S_{l}(0 \leq l \leq 3)$ and ${ }_{3} S_{l}(0 \leq l \leq 1)$ are denoted, respectively, by square, circle, triangle, star and diamond. The black symbol shows that the eigenmode is observed. The eigenmode denoted by other symbols is not excited; the white symbol denotes that the absence of the eigenmode can be explained by its rapid dumping $(\operatorname{Im}(\Omega)>0.0175)$ or weak excitation $(l \geq 7)$, while the gray shaded one denotes that its absence cannot be reasonably explained.

translation $(l=1)$ mode not observed in the solid-sphere system.

In Section 4 of this paper, we attempted explaining the observed signals of a long-period volcanic earthquake by using the eigenmodes of the vibrating fluid sphere under the volcano. It was found that the spectral peaks of the data have very small $\zeta$; for example, the main component of the vibrations possesses $\zeta \simeq 0.003$, implying that it takes more than 50 cycles of vibrations to make its amplitude damped down to $1 / e$. For this reason, it is naturally deduced that those spectral peaks should be explained by the compressional modes in a fluid sphere in our model. We determined the nondimensional parameters which best explain the observation in the following way. First, we assumed that the ratio of the attenuation coefficient to the angular frequency of the primary peak ( $f=1.73 \mathrm{~Hz}$ ) was the same as the value of $\zeta$ that ${ }_{0} L_{1}$ has, because ${ }_{0} L_{1}$ is the compressional mode in fluid of the lowest frequency. If the elastic property in the outer medium is prescribed (we used $\gamma=0.5$ as a nominal value), the number of free parameters decreases to one due to this assumption. Next, we determined a linear relation $f=b \operatorname{Re}(\Omega)$ by enforcing the condition that all the spectral peaks were explained by the compressional modes in fluid with less errors. Then it follows that the relation $f=0.933 \times \operatorname{Re}(\Omega) \mathrm{Hz}$ is a reasonably good approximation to the observation. Last, we assumed that the eigenmode of which the nondimensional attenuation coefficient was larger than 0.02 should be hidden due to its rapid damping. Using the information of the lack of several spectral peaks to which the corresponding eigenmodes exist, we arrived at the conclusion that the observed frequencies and the attenuation coefficients are well explained by specifying $\alpha=3.9 \pm 0.2$ and $\rho=24 \mp 2$.

Of particular interest is how large is the spherical resonator and what is the material inside the sphere? With respect to the size of the spherical resonator, the value of the $\mathrm{P}$ wave velocity in the outer medium is needed. If we adopt that $\alpha_{2}=2500 \mathrm{~m} / \mathrm{s}$ (Sawada et al., 1983), we have $a=\alpha_{2} /(2 \pi b \alpha)=2500 /(2 \times 3.14 \times 0.933 \times 3.9)=110 \mathrm{~m}$; the diameter of the fluid sphere is about $220 \mathrm{~m}$. With respect to the material inside the sphere, its density and pressure give information for estimation. If we adopt that the density in the country rock $\rho_{2}$ is $2200 \mathrm{~kg} / \mathrm{m}^{3}$ and the fluid sphere is $D=800 \mathrm{~m}$ deep beneath the volcano, the estimated value of the fluid density is $\rho_{1}=\rho_{2} / \rho=2200 / 24=92 \mathrm{~kg} / \mathrm{m}^{3}$ and the pressure is $p=\rho_{2} g D=2200 \times 9.8 \times 800=1.7 \times 10^{7}$ $\mathrm{Pa}(=170 \mathrm{~atm})$, where we assumed a hydrostatic balance under a constant gravity $g$. We consider that the fluid sphere is mainly composed of high-pressure vaporized water, firstly because we observed an eventual eruption of vapor at Asama volcano when the earthquake occurred and secondly because the physical property of water at such a high-pressure seems to explain the estimated density of the fluid. Although we don't know the precise form of equation for the state of high-pressure water, there are some grounds to support the vaporized water as a candidate of the fluid material. The temperature and density of steam in saturation at $p=170 \mathrm{~atm}$ are known, respectively, as $630 \mathrm{~K}$ and 135 $\mathrm{kg} / \mathrm{m}^{3}$, and the temperature and pressure of steam in saturation at a density of $\rho_{1}=92 \mathrm{~kg} / \mathrm{m}^{3}$ are, respectively, $610 \mathrm{~K}$ and $144 \mathrm{~atm}$ (Lide, 1993). Provided that the highpressure steam obeys the perfect gas law, its temperature at the state $p=170$ atm and $\rho_{1}=92 \mathrm{~kg} / \mathrm{m}^{3}$ may be narrowed between $630 \times 135 / 92=925 \mathrm{~K}\left(652^{\circ} \mathrm{C}\right)$ and 
Table 2. Our preferred model for the observed long-period event. The nondimensional angular frequencies of the compressional modes in fluid ${ }_{n} S_{l}$ and ${ }_{0} L_{l}$ ) are calculated for $\alpha=3.88, \gamma=0.5$ and $\rho=24$ (listed in the second column). The predicted values (third column) are given by the relations $f=b \cdot \operatorname{Re}(\Omega)$ and $\epsilon=2 \pi b \cdot \operatorname{Im}(\Omega)$, where $b=0.933$. Observed frequency and attenuation coefficient of the spectral peaks are listed in the right column.

\begin{tabular}{|c|c|c|c|c|c|c|}
\hline \multirow[t]{2}{*}{ eigenmode } & \multicolumn{2}{|c|}{ calculated } & \multicolumn{2}{|c|}{ predicted } & \multicolumn{2}{|c|}{ observed } \\
\hline & $\operatorname{Re}(\Omega)$ & $\operatorname{Im}(\Omega)$ & $f[\mathrm{~Hz}]$ & $\epsilon[\mathrm{Hz}]$ & $f[\mathrm{~Hz}]$ & $\epsilon[\mathrm{Hz}]$ \\
\hline${ }_{0} L_{1}$ & 2.071 & 0.00611 & 1.933 & 0.0358 & 1.73 & 0.0318 \\
\hline${ }_{0} L_{2}$ & 3.326 & 0.01443 & 3.104 & 0.0846 & 3.19 & 0.1435 \\
\hline${ }_{0} S_{0}$ & 4.485 & 0.01320 & 4.185 & 0.0774 & 3.65 & 0.0826 \\
\hline${ }_{0} L_{3}$ & 4.491 & 0.02234 & 4.191 & 0.1310 & - & - \\
\hline${ }_{0} L_{4}$ & 5.610 & 0.02587 & 5.235 & 0.1517 & - & - \\
\hline${ }_{0} S_{1}$ & 5.933 & 0.01342 & 5.537 & 0.0787 & 5.79 & 0.0735 \\
\hline${ }_{0} L_{5}$ & 6.707 & 0.02033 & 6.259 & 0.1192 & - & - \\
\hline${ }_{0} S_{2}$ & 7.283 & 0.01316 & 6.797 & 0.0772 & 6.65 & 0.1033 \\
\hline${ }_{1} S_{0}$ & 7.724 & 0.01323 & 7.208 & 0.0776 & 7.14 & 0.1476 \\
\hline${ }_{0} L_{6}$ & 7.797 & 0.01179 & 7.276 & 0.0691 & 7.45 & 0.1273 \\
\hline${ }_{0} S_{3}$ & 8.577 & 0.01380 & 8.004 & 0.0809 & 7.92 & 0.0897 \\
\hline${ }_{0} L_{7}$ & 8.881 & 0.00599 & 8.288 & 0.0351 & - & - \\
\hline${ }_{1} S_{1}$ & 9.204 & 0.01384 & 8.589 & 0.0812 & 8.11 & 0.0792 \\
\hline${ }_{0} S_{4}$ & 9.835 & 0.01534 & 9.178 & 0.0899 & 8.77 & 0.1118 \\
\hline${ }_{0} L_{8}$ & 9.957 & 0.00293 & 9.292 & 0.0172 & - & - \\
\hline${ }_{1} S_{2}$ & 10.610 & 0.01520 & 9.901 & 0.0891 & 10.17 & 0.0669 \\
\hline${ }_{2} S_{0}$ & 10.900 & 0.01206 & 10.172 & 0.0707 & 10.60 & 0.1217 \\
\hline${ }_{0} L_{9}$ & 11.030 & 0.00142 & 10.293 & 0.0083 & - & - \\
\hline${ }_{0} S_{5}$ & 11.070 & 0.01763 & 10.331 & 0.1034 & - & - \\
\hline${ }_{1} S_{3}$ & 11.970 & 0.01619 & 11.171 & 0.0949 & 11.43 & 0.1249 \\
\hline${ }_{0} L_{10}$ & 12.090 & 0.00068 & 11.283 & 0.0040 & - & - \\
\hline${ }_{0} S_{6}$ & 12.270 & 0.02079 & 11.450 & 0.1219 & - & - \\
\hline${ }_{2} S_{1}$ & 12.400 & 0.01232 & 11.572 & 0.0722 & 11.81 & 0.1241 \\
\hline${ }_{0} L_{11}$ & 13.150 & 0.00033 & 12.272 & 0.0019 & - & - \\
\hline${ }_{1} S_{4}$ & 13.290 & 0.01591 & 12.402 & 0.0933 & 12.27 & 0.1129 \\
\hline${ }_{0} S_{7}$ & 13.470 & 0.02519 & 12.570 & 0.1477 & - & - \\
\hline${ }_{2} S_{2}$ & 13.850 & 0.01299 & 12.925 & 0.0762 & - & - \\
\hline${ }_{3} S_{0}$ & 14.070 & 0.01154 & 13.130 & 0.0677 & - & - \\
\hline${ }_{0} L_{12}$ & 14.200 & 0.00016 & 13.252 & 0.0009 & - & - \\
\hline${ }_{1} S_{5}$ & 14.590 & 0.01568 & 13.616 & 0.0919 & 13.61 & 0.0959 \\
\hline${ }_{0} S_{8}$ & 14.650 & 0.03149 & 13.672 & 0.1846 & - & - \\
\hline
\end{tabular}

$610 \times 170 / 144=720 \mathrm{~K}\left(447^{\circ} \mathrm{C}\right)$. It is generally known that the $\mathrm{P}$ wave velocity of gas is proportional to the square root of the absolute temperature and independent of its pressure for a given temperature (Landau and Lifshitz, 1987). Since the sound speed in vapor at $134^{\circ} \mathrm{C}(407 \mathrm{~K})$ is 494 $\mathrm{m} / \mathrm{s}$ (Lide, 1993), the $\mathrm{P}$ wave velocity of the fluid material lies between $\alpha_{1}=494 \times \sqrt{(925 / 407)}=745 \mathrm{~m} / \mathrm{s}$ and $494 \times \sqrt{(720 / 407)}=657 \mathrm{~m} / \mathrm{s}$. These values are comparable to the one $\alpha_{1}=\alpha_{2} / \alpha=2500 / 3.9=640 \mathrm{~m} / \mathrm{s}$, which is estimated by model fitting. Considering the error arising from the parameter estimation, we can say that there is no inconsistency in our conclusion that the resonator is a sphere of diameter $220 \mathrm{~m}$, mainly filled with steam of temperature $\sim 500^{\circ} \mathrm{C}$ and located beneath the vent of the volcano at a depth of $800 \mathrm{~m}$. In this estimation, we used $\rho_{1}=92$ $\mathrm{kg} / \mathrm{m}^{3}, \rho_{2}=2200 \mathrm{~kg} / \mathrm{m}^{3}, \alpha_{1}=640 \mathrm{~m} / \mathrm{s}, \alpha_{2}=2500 \mathrm{~m} / \mathrm{s}$ and $\beta_{2}=1250 \mathrm{~m} / \mathrm{s}$.

The most fundamental assumption we made is that the observed volcanic earthquake occurs due to the resonance of a fluid cavity of which the shape is well approximated by a sphere. Its adequacy cannot be verified unless we find the very cavity under the volcano. However, we have several supporting results which strongly suggest that the observed event is a result of the free oscillations of a spherical fluid cavity under the volcano. Our final result summarized in Fig. 18 and Table 2 shows that the calculated frequencies and attenuation coefficients explain well the observed quantities. The existence of the eigenmodes not observed is a 
natural result due to their high attenuation coefficients.

A problem arising from the present model may be the size of the resonator. We conclude that the diameter of the fluid sphere is $220 \mathrm{~m}$ and it is buried at a depth of $800 \mathrm{~m}$ below the summit crater of Asama volcano. One may think that this configuration is not so realistic to be pictured. However, we consider that the estimated size of the fluid sphere lies within a permissible range because the diameter of the summit crater of Asama is about $300 \mathrm{~m}$ which is larger than the size of the sphere (Fig. 12). There is a good possibility that a cavity exists under the volcano now filled with steam but with magma in the past, which partly causes the present surface features around the summit of Asama.

Whether the fluid sphere really generates the vibrations, as shown in Fig. 1, is not described in this paper. According to the nature of the observed oscillations, it is enough that the excitation of the main spectral peak $(f=1.73 \mathrm{~Hz})$, which is identified as the simple translation mode of a fluid sphere ${ }_{0} L_{1}$, is verified. The selective excitation of the mode of angular order $l=1$ suggests a pulsative force acting along one axis. If the vapor flows into the cavity at one side or escapes out of it at one side, the translation mode may be effectively excited.

The present fluid-sphere model is one candidate and, of course, there could be other models to explain the observed long-period event. In particular, Kumagai and Chouet (1999) claim that a thin crack with length of $100 \mathrm{~m}$ filled with ash-laden gases can explain the long-period event observed at the Kusatsu-Shirane volcano whose waveform and the long-lasting features are very similar to those of Asama. A fluid-filled crack may thus provide an alternative to our fluid-sphere model.

Three components of ground motion could give us information about the source of the long-period event. In this application, however, the velocity data, except for the up-down component, seemed to have considerable errors. Using all the components of the ground motion, we could obtain more details of the source mechanism.

It is worth noting that the imaginary part of the eigenfrequency changes sensitively with the change of the nondimensional parameters in our model. Therefore, measurement of attenuation coefficients will be of critical importance in determining the source parameters from the seismic data. In this respect, we have to pay attention to the seismic character in the volcanic region. For example, seismic signals may be contaminated through the effect of porosity of crust materials.

In this paper, we made an attempt to completely describe the nature of vibrations of a fluid sphere in an infinite elastic medium. We believe that this study provides a reference to interpret seismic data of long-period events.

Acknowledgments. We appreciate comments and suggestions by Dr. Bernard Chouet and Dr. Tatsuhiko Hara which greatly improved this paper.

\section{References}

Aki, K. and P. G. Richards, Quantitative Seismology, vol. 1, W. H. Freeman \& Co., New York, 1980.

Chouet, B. A., Excitation of a buried magmatic pipe, J. Geophys. Res., 90, 1881-1893, 1985

Chouet, B. A., Long-period volcano seismicity: its source and use in eruption forecasting, Nature, 380, 309-316, 1996.

Chouet, B. A., Resonance of a fluid-driven crack: Radiation properties and implications for the source of long-period events and harmonic tremor, J. Geophys. Res., 93, 4375-4400, 1988.

Crosson, R. S. and D. A. Bame, A spherical source model for low frequency volcanic earthquakes, J. Geophys. Res., 90, 10237-10247, 1985.

Ferrick, M. G., A. Qamer, and W. St. Lawrence, Source mechanisms of volcanic tremor, J. Geophys. Res., 87, 8675-8683, 1982.

Fujita, E., Y. Ida, and J. Oikawa, Eigen oscillation of a fluid sphere and source mechanism of harmonic volcanic tremor, J. Volcano. Geotherm. Res., 69, 365-378, 1995.

Gómez, M., D. M., and R. A. Torres C., Unusual low-frequency volcanic seismic events with slowly decaying coda waves observed at Galeras and other volcanoes, J. Volcano. Geotherm. Res., 77, 173-193, 1997.

Julian, B. R., Volcanic tremor: Nonlinear excitation by fluid flow, J. Geophys. Res., 99, 11859-11877, 1994.

Kedar, S., B. Sturtevant, and H. Kanamori, The origin of harmonic tremor at Old Faithful geyser, Nature, 379, 708-711, 1996.

Kumagai, H. and B. A. Chouet, The complex frequencies of long-period seismic events as probes of fluid composition beneath volcanoes, Geophys. J. Int., 138, F7-F12, 1999.

Landau, L. D. and E. M. Lifshitz, Theory of Elasticity, Pergamon Press, Oxford, 3rd edition, 1986.

Landau, L. D. and E. M. Lifshitz, Fluid Mechanics, Pergamon Press, Oxford, 2nd edition, 1987

Lide, D. R., Handbook of Chemistry and Physics, CRC Press, 74th edition, 1993.

McNutt, S. R., Volcanic tremor, in Encyclopedia of Earth System Science, edited by W. A. Nierenberg, Vol. 4, pp. 417-425, Academic Press, 1992.

Nishimura, Y. and H. Yamashita, Earthquake swarm at Meakan volcano in March, 1982, Geophys. Bull. Hokkaido Univ., 41, 65-76, 1982 (in Japanese with English abstract).

Sawada, M., N. Gyoda, N. Osada, E. Koyama, H. Tsui, T. Kagiyama, and T. Miyazaki, P wave velocity structure of Asama volcano, Bull. Volcanol. Soc. Japan. Ser. 2, 28, 301-304, 1983 (in Japanese with English abstract).

Tsuji, H., N. Gyoda, E. Koyama, J. Oikawa, and Y. Ida, Seismic observation at the summit of Asama volcano, Technical Research Report of Earthquake Research Institute, University of Tokyo, 2, 135-140, 1998 (in Japanese).

A. Sakuraba (e-mail: sakuraba@geoph.s.u-tokyo.ac.jp), J. Oikawa (email: oikawa@eri.u-tokyo.ac.jp), and Y.Imanishi(e-mail: imanishi@ori.utokyo.ac.jp) 